\title{
Detection of Quasi-Static Electric Fields Radiated by Electrically Small Emitters
}

David A. Hill

Kenneth H. Cavcey 



\section{Detection of Quasi-Static Electric Fields Radiated by Electrically Small Emitters}

David A. Hill

Kenneth H. Cavcey

Electronics and Electrical Engineering Laboratory

Radio-Frequency Technology Division

June 2000

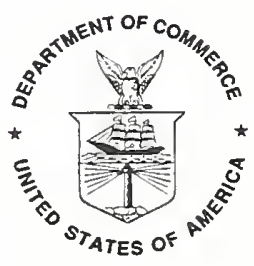

U.S. Department of Commerce William M. Daley, Secretary

Technology Administration

Dr. Cheryl L. Shavers, Under Secretary of Commerce for Technology 


\section{National Institute of Justice}

Jeremy Travis

Director

The technical effort to develop this standard was conducted under Interagency Agreement No. 94-IJ-R-004, Project No. 97-017CTT

This report was prepared by the Office of Law

Enforcement Standards (OLES) of the

National Institute of Standards and Technology (NIST)

under the direction of A. George Lieberman, Program Manager for Communications Systems, and Kathleen M. Higgins, Director of OLES. The research resulting in this report was performed by David A. Hill and Kenneth $\mathrm{H}$. Cavcey of the NIST Boulder laboratories, Electronics and Electrical Engineering Laboratory, Radio Frequency Technology Division. The work was sponsored by the National Institute of Justice, David G. Boyd, Director, Office of Science and Technology. 


\section{ABOUT THE LAW ENFORCEMENT AND CORRECTIONS STANDARDS AND TESTING PROGRAM}

The Law Enforcement and Corrections Standards and Testing Program is sponsored by the Office of Science and Technology of the National Institute of Justice (NIJ), U.S. Department of Justice. The program responds to the mandate of the Justice System Improvement Act of 1979, which created NIJ and directed it to encourage research and development to improve the criminal justice system and to disseminate the results to Federal, State, and local agencies.

The Law Enforcement and Corrections Standards and Testing Program is an applied research effort that determines the technological needs of justice system agencies, sets minimum performance standards for specific devices, tests commercially available equipment against those standards, and disseminates the standards and the test results to criminal justice agencies nationally and internationally.

The program operates through:

The Law Enforcement and Corrections Technology Advisory Council (LECTAC) consisting of nationally recognized criminal justice practitioners from Federal, State, and local agencies, which assesses technological needs and sets priorities for research programs and items to be evaluated and tested.

The Office of Law Enforcement Standards (OLES) at the National Institute of Standards and Technology, which develops voluntary national performance standards for compliance testing to ensure that individual items of equipment are suitable for use by criminal justice agencies. The standards are based upon laboratory testing and evaluation of representative samples of each item of equipment to determine the key attributes, develop test methods, and establish minimum performance requirements for each essential attribute. In addition to the highly technical standards, OLES also produces technical reports and user guidelines that explain in nontechnical terms the capabilities of available equipment.

The National Law Enforcement and Corrections Technology Center (NLECTC), operated by a grantee, which supervises a national compliance testing program conducted by independent laboratories. The standards developed by OLES serve as performance benchmarks against which commercial equipment is measured. The facilities, personnel, and testing capabilities of the independent laboratories are evaluated by OLES prior to testing each item of equipment, and OLES helps the NLECTC staff review and analyze data. Test results are published in Equipment Performance Reports designed to help justice system procurement officials make informed purchasing decisions.

Publications are available at no charge from the National Law Enforcement and Corrections Technology Center. Some documents are also available online through the Internet/World Wide Web. To request a document or additional information, call $800-248-2742$ or 301-519-5060, or write:

National Law Enforcement and Corrections Technology Center

P.O. Box 1160

Rockville, MD 20849-1160

E-mail: asknlectc@nlectc.org

World Wide Web address: http://www.nlectc.org

The National Institute of Justice is a component of the Office of Justice Programs, which also includes the Bureau of Justice Assistance, Bureau of Justice Statistics, Office of Juvenile Justice and Delinquency Prevention, and the Office for Victims of Crime. 



\section{CONTENTS}

Page

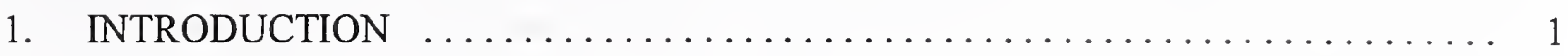

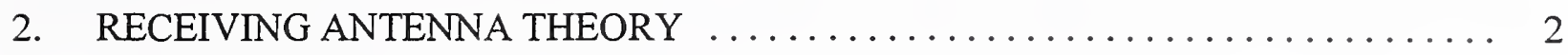

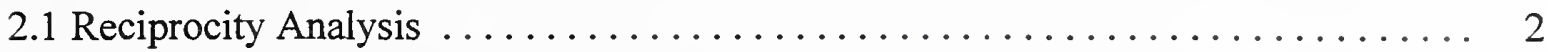

2.2 Induced EMF Method $\ldots \ldots \ldots \ldots \ldots \ldots \ldots \ldots \ldots \ldots \ldots \ldots \ldots \ldots \ldots \ldots$

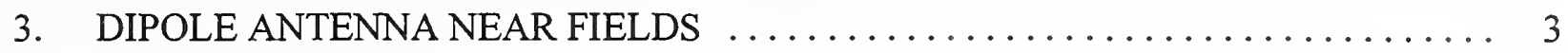

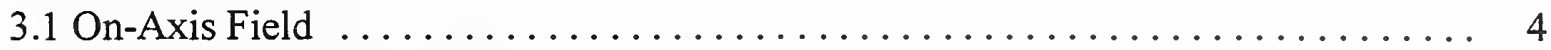

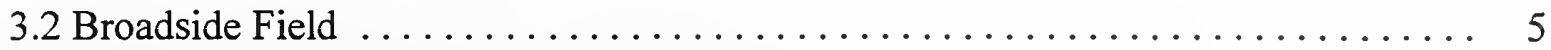

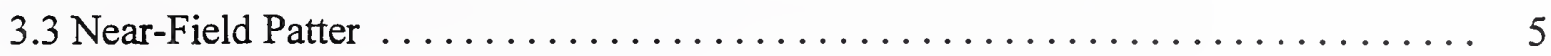

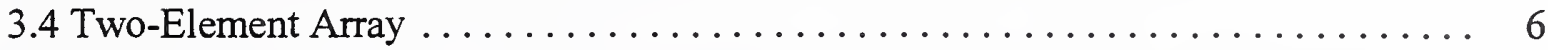

4. MONOPOLE ANTENNA NEAR FIELDS $\ldots \ldots \ldots \ldots \ldots \ldots \ldots \ldots \ldots \ldots$

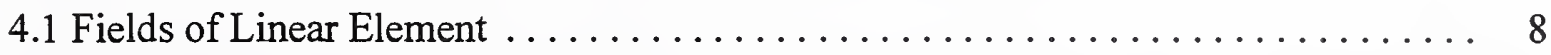

4.2 Ground Screen Currents and Fields ......................... 10

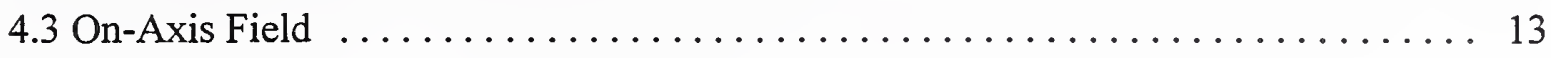

4.4 Comparison of Front and Back Fields . . . . . . . . . . . . . . . 14

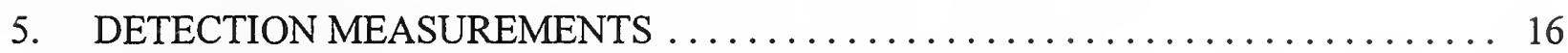

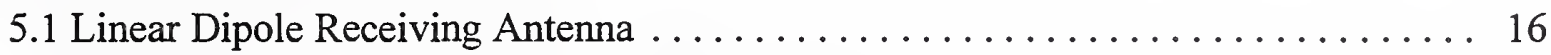

5.2 Monopole Receiving Antenna $\ldots \ldots \ldots \ldots \ldots \ldots \ldots \ldots \ldots \ldots \ldots \ldots \ldots \ldots$

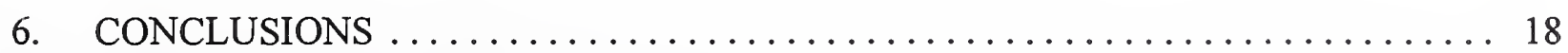

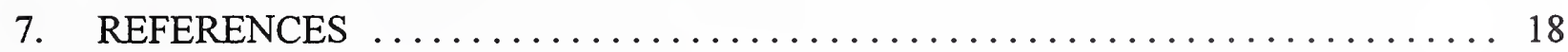

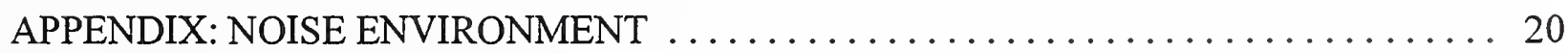




\section{FOREWORD}

The Office of Law Enforcement Standards (OLES) of the National Institute of Standards and Technology (NIST) furnishes technical support to the National Institute of Justice (NIJ) program to strengthen law enforcement and criminal justice in the United States. OLES's function is to conduct research that will assist law enforcement and criminal justice agencies in the selection and procurement of quality equipment.

OLES is: (1) Subjecting existing equipment to laboratory testing and evaluation, and (2) conducting research leading to the development of several series of documents, including national standards, user guides, and technical reports.

This document covers research conducted by OLES under the sponsorship of the NIJ. Additional reports as well as other documents are being issued under the OLES program in the areas of protective clothing and equipment, communications systems, emergency equipment, investigative aids, security systems, vehicles, weapons, and analytical techniques and standard reference materials used by the forensic community.

Technical comments and suggestions concerning this report are invited from all interested parties. They may be addressed to the Office of Law Enforcement Standards, National Institute of Standards and Technology, Gaithersburg, MD 20899-8102.

David G. Boyd, Director

Office of Science and Technology

National Institute of Justice 


\section{PREFACE}

This is the third and final report in a three-year project on Detection of Electronic Bomb Detonators, and it covers the period from January 1999 through March 2000. The project is sponsored by the National Institute of Justice, David G. Boyd, Director, Office of Science and Technology. The research was performed by the NIST Radio-Frequency Technology Division and was monitored by A. George Lieberman, Program Manager for Communications Systems and Kathleen M. Higgins, Director of the Office of Law Enforcement Standards (OLES).

The first report on this project covered the theory of separating the fields of interest (from electronic timers) from undesired noise fields from external sources by using the techniques of spherical, near-field scanning as developed at NIST:

Hill, D.A. Spherical-wave characterization of interior and exterior electromagnetic sources. Natl. Inst. Stand. Technol. NISTIR 5072; December 1997.

The second report on this project covered the theory and measurement techniques for determining the dipole moments of weak, electrically small emitters. The measurement technique made use of a transverse electromagnetic (TEM) cell, and it was used to determine the three orthogonal electric dipole moments of two electronic timers for frequencies from $200 \mathrm{kHz}$ to $8 \mathrm{MHz}$. The magnetic dipole moments were found to be too small to measure. The practical conclusions were that electronic timers of the type that could be used in bomb detonators do radiate weak electric fields that can be measured using TEM cells that have been well characterized at NIST:

Hill, D.A.; Cavcey, K.H. Dipole moments of weak, electrically small emitters from TEM-cell measurements. Natl. Inst. Stand. Technol. NISTIR 5079; December 1998.

The purposes of this report are (a) to present the theory for near-field detection of quasistatic electric fields produced by electrically small emitters and (b) to demonstrate detection of these fields with linear dipole and monopole antennas. These antenna types were chosen because they respond well to electric fields and they are sufficiently portable that they could be used in a search operation where the location of the small radiator (electronic timer) is unknown. Successful detection measurements of timer electric fields were achieved at a frequency of 200 $\mathrm{kHz}$ with both antenna types, but the detection range $(10$ to $20 \mathrm{~cm})$ is much too short to be used in a search application. This small detection range falls below the theoretical prediction made from previous TEM-cell characterization of electronic timer radiation. One of the main reasons is that the background noise fields were larger than expected from published values. The unexpectedly large background noise fields required us to narrow the measurement bandwidth, hence reducing the desired signal and increasing the measurement uncertainty. 


\section{-}




\title{
DETECTION OF QUASI-STATIC ELECTRIC FIELDS \\ RADIATED BY ELECTRICALLY SMALL EMITTERS
}

\author{
David A. Hill and Kenneth H. Cavcey \\ Radio Frequency Technology Division \\ National Institute of Standards and Technology \\ Boulder, Colorado 80303
}

This report presents the theory for near-field detection of quasi-static electric fields produced by electrically small emitters that radiate as small electric dipoles. The intended application is the detection of electronic timers of the type that could be used in bomb detonators. The search antenna could be either a linear dipole or a monopole antenna with a circular ground plane. Reciprocity is used to show that the induced open-circuit voltage is proportional to the electric field of the search antenna at the dipole source location when the search antenna is used in the transmitting mode. Comparison detection measurements have been made at a frequency of $200 \mathrm{kHz}$, and the measured open-circuit voltage falls below the theoretical prediction. Also, the background noise fields are higher than expected from published values. Consequently, the detection range is much shorter (10 to $20 \mathrm{~cm}$ ) than expected, and the method does not look promising for timer search applications.

Key words: dipole moment; electrically small emitter; linear dipole antenna; monopole antenna; noise spectral density; quasi-static electric field; reciprocity.

\section{INTRODUCTION}

In a previous report [1], we showed that electronic timers radiate very weak quasi-static electric fields in the same manner as does an electric dipole source. The magnitudes of the electric dipole moments at low frequencies were determined using a transverse electromagnetic (TEM) cell, and no magnetic dipole moments were detectable [1]. Spherical scanning techniques [2] could be used to determine the electric dipole moments, but in a search operation we do not expect to be able to surround the timer with an array of field probes. In searching for an electronic timer of the type that could be used in a bomb detonator, a single portable antenna is a more practical search device.

The purposes of this report are (a) to present the theory for near-field detection of quasistatic electric fields $[3,4]$ produced by electrically small emitters and (b) to demonstrate detection of these electric fields with linear dipole and monopole antennas. Reciprocity is used in the general receiving formulation in Section 2, and the mathematical details for dipole and monopole antennas are given in Sections 3 and 4. Successful timer detection measurements with dipole and monopole antennas are described in Section 5, but the detection range is found to be much too short for practical search applications. 


\section{RECEIVING ANTENNA THEORY}

In general the analysis of the response of a receiving antenna to the nonuniform field of a near-field source is very complicated. However, for simple linear antennas at low frequencies, reciprocity and the induced electromotive force (EMF) method can be used to obtain good approximations for the open-circuit voltage induced in the receiving antenna.

\subsection{Reciprocity Analysis}

Reciprocity analysis is based on two source and field states that we denote as states 1 and 2. Since we are interested in the case of detecting electric dipole radiation of electronic timers with wire antennas, only electric currents and fields are required in the formulation. So the reciprocity integral relationship can be written [5]

$$
\iiint_{V} J_{2}(\boldsymbol{r}) \cdot \boldsymbol{E}_{1}(\boldsymbol{r}) \mathrm{d} V=\iiint_{V} J_{1}(\boldsymbol{r}) \cdot \boldsymbol{E}_{2}(\boldsymbol{r}) \mathrm{d} V,
$$

where $J_{1}$ and $E_{1}$ are the electric current density and electric field of state 1 and $J_{2}$ and $E_{2}$ are the electric current density and electric field of state 2 . The time dependence of the currents and fields is $\exp (j \omega t)$. We assume that the currents radiate in free space so that no boundary surface integrals are required in eq (1).

Consider now the geometry of a linear dipole antenna and a small emitter as shown in figure 1 . In state 1 , the dipole antenna is radiating, and the current density $J_{1}$ can be written

$$
J_{1}(r)=\hat{z} \delta(x) \delta(y) I_{1}(z),
$$

where $\hat{z}$ is a unit vector along the axis of the antenna, $\delta$ is the Dirac delta function, and $I_{1}$ is the antenna current. In state 2 , the emitter is radiating, and the current density $J_{2}$ can be written

$$
J_{2}(r)=j \omega p_{e} \delta\left(r-r_{2}\right)
$$

where $\boldsymbol{p}_{e}$ is the electric dipole moment of the emitter located at $\boldsymbol{r}_{2}$. For later results, it is convenient to write the vector dipole moment in terms of its rectangular components:

$$
\boldsymbol{p}_{e}=\hat{\boldsymbol{x}} p_{e x}+\hat{\boldsymbol{y}} p_{e y}+\hat{z} p_{e z} .
$$

If we substitute eqs (2) and (3) into eq (1) and perform the integrations, we obtain

$$
j \omega p_{e} \cdot E_{1}\left(r_{2}\right)=\int_{-h}^{h} E_{2 z}(z) I_{1}(z) \mathrm{d} z,
$$

where $h$ is the half length of the dipole antenna. Actually, the form in eq (5) applies equally to other linear antennas with only a change in the limits of integration on the right side. Except for 
the thin-wire approximation, eq (5) is exact as written. However, we will later introduce approximate expressions for the current $I_{1}$ and the resultant electric field $E_{1}$.

\subsection{Induced EMF Method}

The induced EMF method [6] provides a convenient method for determining the induced open-circuit voltage $V_{o c}$ of a linear antenna in terms of its transmitting current distribution and the incident electric field. For the geometry in figure 1, $V_{o c}$ can be written [6]

$$
V_{o c}=\frac{-1}{I_{1}(0)} \int_{-h}^{h} E_{2 z}(z) I_{1}(z) \mathrm{d} z
$$

If we substitute eq (5) into eq (6), we obtain the desired result for $V_{o c}$ :

$$
V_{o c}=-\frac{j \omega}{I_{1}(0)} p_{e} \cdot E_{1}\left(r_{2}\right) .
$$

In an actual evaluation of eq $(7)$, the factor $I_{1}(0)$ will cancel because $E_{1}$ is proportional to $I_{1}(0)$.

The advantage of eq (7) is that the received voltage can be determined in terms of the transmitted electric field $\boldsymbol{E}_{1}$ evaluated at the emitter location $\boldsymbol{r}_{2}$. This feature of reciprocity is well known for the far-field transmitting and receiving patterns of antennas [7], but it is equally applicable to near-field applications. Since the orientation of the emitter will not be known and the measured rectangular components of $p_{e}$ are nearly equal [1], a good measure of the effectiveness of an antenna in detecting a timer at a location $\boldsymbol{r}_{2}$ is $\left|\boldsymbol{E}_{1}\left(\boldsymbol{r}_{2}\right) / I_{1}(0)\right|$. This quantity has units of $\Omega / \mathrm{m}$, and the quantity $j \omega p_{e}$ has units of $A \cdot m$.

The induced EMF method can also be used to determine the input impedance of linear antennas [6]. When the dipole length $2 h$ is small compared to the free-space wavelength $\lambda$, the imput impedance is primarily a capacitive reactance $\left(j \omega C_{d}\right)^{-1}$. The dipole capacitance $C_{d}$ is given by $[8]$

$$
C_{d}=\frac{\pi \varepsilon_{0} h}{\ln (2 h / d)-1}
$$

where $\varepsilon_{0}$ is the permittivity of free space and $d$ is the wire diameter. The approximate equivalent circuit is shown in figure 2 , where the small radiation resistance of the dipole antenna is neglected.

\section{DIPOLE ANTENNA NEAR FIELDS}

For thin dipole antennas with a length not exceeding a half wavelength, the current distribution $I(z)$ can be approximated as sinusoidal [6]: 


$$
I(z)=I_{0} \frac{\sin [k(h-|z|)]}{\sin (k h)},|z|<h,
$$

where $I_{0}$ equals the current at the feedpoint $I(0), k=\omega \sqrt{\mu_{0} \varepsilon_{0}}$, and $\mu_{0}$ is the magnetic permeability of free space. For our low-frequency application, the dipole antenna is electrically short $(k h<<1)$ and eq (9) reduces to the following triangular distribution

$$
I(z) \approx I_{0}\left(1-\frac{|z|}{h}\right), \quad|z|<h .
$$

The nonzero electric field components, $E_{\rho}$ and $E_{z}$, and magnetic field component $H_{\phi}$ for the dipole current distribution in eq (9) are well known [6]. In the quasi-static approximation, we assume that both the dipole length and the distance to the field point are electrically small $(k r<<1)$. In this case, the current distribution in eq $(10)$ generates the following electric field components:

$$
E_{\rho}=\frac{-I_{0}}{4 \pi j \omega \varepsilon_{0} h \rho}\left(\frac{z-h}{r_{1}}-\frac{2 z}{r}+\frac{z+h}{r_{2}}\right)
$$

and

$$
E_{z}=\frac{I_{0}}{4 \pi j \omega \varepsilon_{0} h}\left(\frac{1}{r_{1}}-\frac{2}{r}+\frac{1}{r_{2}}\right)
$$

where $r=\sqrt{\rho^{2}+z^{2}}, r_{1}=\sqrt{\rho^{2}+(z-h)^{2}}$, and $r_{2}=\sqrt{\rho^{2}+(z+h)^{2}}$. The geometry is shown in figure 3. The main difference between the quasi-static results and the general results [6] is that there are no exponential propagation factors in eqs (11) and (12). Even though eqs (11) and (12) are quasi-static approximations, we use " $=$ " rather than " $\approx$ " here and throughout the rest of this report whenever the results are exact within the quasi-static regime. A similar expression could be derived for the magnetic field $H_{\phi}$, but is not needed here.

\subsection{On-Axis Field}

In a search application, the electric field on the $z$ axis $(\rho=0)$ is important because the axial field $E_{z}$ has its largest magnitude there. The $\rho$ component of the field $E_{\rho}$ is zero on the axis, and the $z$ component on the axis can be obtained directly from eq (12):

$$
\left.E_{z}\right|_{\rho=0}=\frac{I_{0}}{4 \pi j \omega \varepsilon_{0} h}\left(\frac{1}{|z-h|}-\frac{2}{|z|}+\frac{1}{|z+h|}\right) .
$$

Equation (13) can be simplified by combining terms: 


$$
\left.E_{z}\right|_{\rho=0}=\frac{I_{0} h}{2 \pi j \omega \varepsilon_{0}|z|\left(z^{2}-h^{2}\right)} .
$$

Equation (14) is actually valid for all values of $z$, but is physically meaningful only for $|z|>h$.

For large values of $z^{2} / h^{2}$, eq (14) simplifies to the expected inverse distance-cubed dependence:

$$
\left.E_{z}\right|_{\rho=0} \approx \frac{I_{0} h}{2 \pi j \omega \varepsilon_{0}|z|^{3}}
$$

This agrees with the quasi-static field of a Hertzian dipole $[3,4]$ of moment $\left(I_{0} h\right) /(j \omega)$. It is even in $z$ as expected for a symmetrical dipole antenna. The practical implication of eq (15) in a search application is that the received signal will fall off as distance cubed when the emitter is located more than a few dipole lengths from the dipole.

\subsection{Broadside Field}

The broadside field $(z=0)$ is also of interest in search applications because it is possible to move the center of the dipole quite close to the emitter in the $z=0$ plane. The $\rho$ component is zero for $z=0$, and the $z$ component can be obtained directly from eq (12):

$$
\left.\dot{E}_{z}\right|_{z=0}=\frac{I_{0}}{2 \pi j \omega \varepsilon_{0} h}\left(\frac{1}{\sqrt{\rho^{2}+h^{2}}}-\frac{1}{\rho}\right) .
$$

For small $\rho$, the field varies as inverse distance.

For large values of $\rho^{\prime} h$, the inverse distance terms cancel, and the field takes on the expected inverse distance-cubed dependence:

$$
\left.E_{z}\right|_{z=0} \approx \frac{-I_{0} h}{4 \pi j \omega \varepsilon_{0} \rho^{3}} .
$$

This result is similar to the on-axis result in eq (15) except that the sign is reversed and the magnitude is reduced by a factor of 2 . This is quite different from the radiation field where the maximum field is in the broadside direction and a null exists on the $z$ axis.

\subsection{Near-Field Pattern}

Equations (11) and (12) give expressions for $E_{\rho}$ and $E_{z}$ for general observation points. For $r / h>>1$, these equations simplify to 


$$
E_{\rho} \approx \frac{I_{0} h}{2 \pi j \omega \varepsilon_{0} r^{3}}\left(\frac{3}{2} \sin \theta \cos \theta\right)
$$

and

$$
E_{z} \approx \frac{I_{0} h}{2 \pi j \omega \varepsilon_{0} r^{3}}\left(\cos ^{2} \theta-\frac{1}{2} \sin ^{2} \theta\right)
$$

where the polar angle $\theta=\tan ^{-1}(\rho / z)$. The geometry is shown in figure 4 .

Equations (18) and (19) have the form of $r^{-3}$ times a function of $\theta$. As indicated in Section 3.2, the magnitude of the electric field is the important quantity in near-field detection. Using eqs (18) and (19), we can write the magnitude of the electric field $|E|$ in the following manner

$$
|\boldsymbol{E}|=\sqrt{\left|E_{\rho}\right|^{2}+\left|E_{z}\right|^{2}} \approx\left(\frac{I_{0} h}{2 \pi \omega \varepsilon_{0} r^{3}}\right) P(\theta)
$$

where

$$
P(\theta)=\sqrt{\cos ^{2} \theta+\frac{1}{4} \sin ^{2} \theta}
$$

$P(\theta)$ can be thought of as the quasi-static field pattern of a linear dipole. It has a maximum value of 1 at $\theta=0$ and $\pi$ and a minimum value of $1 / 2$ at $\theta=\pi / 2$, and it has no nulls. This is very different from the $\sin \theta$ radiation pattern of the same dipole, which has a maximum at $\theta=\pi / 2$ and nulls at $\theta=0$ and $\pi$. Both patterns are shown in figure 5 .

\subsection{Two-Element Array}

The usual advantages of antenna arrays [9] are high gain and the possibility of beam scanning. However, normal array design requires a significant electrical spacing between elements (on the order of a half wavelength) to produce a directive array pattern. Since that is not possible in our quasi-static search application, it is not clear that arrays offer any advantage.

However, one possible advantage of a simple two-element array is the cancellation of noise due to distant sources. (See the Appendix for expected noise characteristics.) The idea is that the near-field of an emitter is very nonuniform because of its inverse distance-cubed dependence, but the field of a distant noise source is fairly uniform (nearly a plane wave). So a two-element array with $180^{\circ}$ phasing will nearly cancel the uniform noise signal, but should produce less cancellation of the emitter signal. This type of array is sometimes called a gradiometer [10] because it responds primarily to the gradient of the field.

The two-element, colinear array we consider is shown in figure 6 . The dipole centers are separated by a distance $s$, and the terminal currents are $+I_{0}$ and $-I_{0}$. We again follow the reciprocity formulation of Section 2 so that we need to consider only the transmitting case. We 
can use the field expressions in eqs (11) and (12) plus superposition to obtain the following expressions for the array fields:

$$
\begin{aligned}
E_{\rho}=\frac{-I_{0}}{4 \pi j \omega \varepsilon_{0} h \rho} & \left\{\left[\frac{z-h-s / 2}{r_{1+}}-\frac{2(z-s / 2)}{r_{+}}+\frac{z+h-s / 2}{r_{2+}}\right]\right. \\
& \left.-\left[\frac{z-h+s / 2}{r_{1-}}-\frac{2(z+s / 2)}{r_{-}}+\frac{z+h+s / 2}{r_{2-}}\right]\right\}
\end{aligned}
$$

and

$$
E_{z}=\frac{I_{0}}{4 \pi j \omega \varepsilon_{0} h}\left[\left(\frac{1}{r_{1+}}-\frac{2}{r_{+}}+\frac{1}{r_{2+}}\right)-\left(\frac{1}{r_{1-}}-\frac{2}{r_{-}}+\frac{1}{r_{2-}}\right)\right],
$$

where $r_{1+}=\sqrt{\rho^{2}+(z-h-s / 2)^{2}}, r_{+}=\sqrt{\rho^{2}+(z-s / 2)^{2}}, r_{2+}=\sqrt{\rho^{2}+(z+h-s / 2)^{2}}$,

$$
r_{1-}=\sqrt{\rho^{2}+(z-h+s / 2)^{2}}, r_{-}=\sqrt{\rho^{2}+(z+h+s / 2)^{2}} \text {, and } r_{2-}=\sqrt{\rho^{2}+(z+h+s / 2)^{2}} \text {. }
$$

The terms in (22) and (23) tend to cancel each other, so that the field strength tends to be reduced over that of a single element. One way to see this clearly is to derive an expression for the field magnitude for large $r$ and to compare it with the result for a single dipole in eq (20). For the condition $r /(s+h) \gg 1$, the following appoximation can be obtained from eqs (22) and (23):

$$
|E|=\sqrt{\left|E_{\rho}\right|^{2}+\left|E_{z}\right|^{2}} \approx\left[\frac{I_{0} h P(\theta)}{2 \pi \omega \varepsilon_{0} r^{3}}\right]\left[\frac{3 s|\cos \theta|}{r}\right]
$$

where $P(\theta)$ is given by eq (21). Comparing eqs (20) and (24), we see that the array field is reduced because the second factor on the right side of eq (24) is small $(s / r<<1)$.

To determine the amount of noise cancellation achieved by a two-element array, it is more convenient to consider the receiving case for plane-wave incidence. The geometry for a plane-wave noise field incident on the array is shown in figure 7. For incidence at an angle $\theta$ to the $z$ axis, the open circuit voltage is the difference of two terms with slightly different phases:

$$
V_{o c}=L_{e f f} E_{n \theta} \sin \theta\left[\exp \left(j k \frac{s}{2} \cos \theta\right)-\exp \left(-j k \frac{s}{2} \cos \theta\right)\right],
$$

where $L_{\text {eff }}$ is the effective length of one dipole and $E_{n \theta}$ is the $\theta$ component of the electric field of the noise. For electrically short dipoles, the effective length is approximately half the physical length $\left(L_{\text {eff }} \approx h\right)$. The exponentials in $(25)$ can be approximated to yield 


$$
V_{o c} \approx h E_{n \theta} \sin \theta R_{n},
$$

where $R_{n}=j k s \cos \theta$. We can consider $R_{n}$ to be a noise reduction factor resulting from the cancellation in the array. For $\theta=0$, total cancellation occurs, and $R_{n}=0$. Even for other angles, the cancellation is effective because $k s$ is small. For example, consider the case where $s=1 \mathrm{~m}$ and the frequency is $200 \mathrm{kHz}$ (wavelength $\lambda=1.5 \times 10^{3} \mathrm{~m}$ ). Then $k s$ is

$$
k s=\frac{2 \pi}{\lambda} s=\frac{2 \pi}{1.5 \times 10^{3} \mathrm{~m}} 1 \mathrm{~m} \approx 4.19 \times 10^{-3} .
$$

Hence this array would provide significant noise cancellation at $200 \mathrm{kHz}$, the frequency where timer emissions are significant [1].

\section{MONOPOLE ANTENNA NEAR FIELDS}

Linear dipole antennas have the advantages that they are simple, well characterized, and good receivers of electric fields as shown in the previous section. Their main disadvantage in a search application is that they do not have a single main beam. The quasi-static pattern in figure 5 shows broad peaks off both ends $\left(\theta=0^{\circ}\right.$ and $\left.180^{\circ}\right)$. This means that location of an emitter (timer) would have to be made by moving the search dipole and observing a stronger signal as the dipole approached the emitter. It would be faster and more convenient if the search antenna had some near-field directivity and a single pattern maximum. Since arrays are not promising for near-field directivity, another approach is needed.

A monopole antenna with a circular ground plane, as shown in figure 8, is a logical candidate because it also responds well to electric fields. In the forward half space $(z>0)$, the fields are nearly the same as those of a dipole. In the backward half space $(z<0)$, the ground screen shields and reduces the fields. (For an infinite radius $a$, the fields are zero for negative $z$ and by image theory are the same as those of a dipole for positive $z$.) The purpose of this section is to derive expressions for the quasi-static electric field based on approximations for the monopole current and ground screen current available in the literature.

\subsection{Fields of Linear Element}

Since the sinusoidal current assumption is also a good approximation for the current $I_{m}(z)$ on the vertical monopole element [11], we can again use the linear current approximation of eq (10) for the current distribution:

$$
I_{m}(z) \approx I_{0}\left(1-\frac{z}{h}\right), \quad 0 \leq z \leq h
$$

where $I_{0}$ is the base current. Usually the electric and magnetic fields of linear current distributions are derived from the magnetic vector potential [6], but we chose to use the scalar potential to derive the quasi-static electric field because it will prove to be simpler in deriving the electric field of the ground screen currents. 
The quasi-static electric field can be derived from the gradient of the scalar potential $\Phi$ :

$$
E(r)=-\nabla \Phi(r)
$$

The scalar potential is determined by the charge distribution, rather than the current distribution. In general we can determine the volume charge distribution $q_{v}$ from the volume current density $J$ by means of the continuity eq [4]

$$
\nabla \cdot J(r)=-j \omega q_{v}(r)
$$

The linear charge distribution $q_{l}$ on the monopole can be obtained by specializing eq (30) to the linear current distribution in eq (28):

$$
q_{l}(z)=\frac{-1}{j \omega} \frac{\partial I_{m}(z)}{\partial z}=\frac{I_{0}}{j \omega h}, \quad 0<z \leq h .
$$

Strictly speaking, there is a point charge equal to $-I_{0} /(j \omega)$ at $z=0$ due to the discontinuity in $I_{m}$ at $z=0$. However, we chose to neglect it because it will be cancelled by an equal and opposite point charge at the center of the ground screen. So the total electric field due to the total electric charge on the linear monopole and the circular ground screen will be correct.

The general expression for the scalar potential in terms of an integral of volume charge can be specialized to the following line integral

$$
\Phi=\frac{1}{4 \pi \varepsilon_{0}} \int_{0}^{h} \frac{q_{l}\left(z^{\prime}\right)}{R} \mathrm{~d} z^{\prime}=\frac{I_{0}}{4 \pi j \omega \varepsilon_{0} h} \int_{0}^{h} \frac{d z^{\prime}}{R}
$$

where $R=\sqrt{\left(z-z^{\prime}\right)^{2}+\rho^{2}}$. The geometry is shown in figure 9. The integral in eq (32) can be evaluated analytically to obtain

$$
\Phi=\frac{I_{0}}{4 \pi j \omega \varepsilon_{0} h} \ln \left[\frac{\sqrt{(h-z)^{2}+\rho^{2}}+h-z}{\sqrt{z^{2}+\rho^{2}}-z}\right] .
$$

The electric field components, $E_{m \rho}$ and $E_{m z}$, due to the monopole are obtained from the gradient of eq (33):

$$
E_{m \rho}=-\frac{\partial \Phi}{\partial \rho}=\frac{-I_{0}}{4 \pi j \omega \varepsilon_{0} h \rho}\left(\frac{z-h}{r_{1}}-\frac{z}{r}\right)
$$

and 


$$
E_{m z}=-\frac{\partial \Phi}{\partial z}=\frac{I_{0}}{4 \pi \omega \varepsilon_{0} h}\left(\frac{1}{r_{1}}-\frac{1}{r}\right)
$$

These electric field results are consistent with those of the full dipole in eqs (11) and (12).

\subsection{Ground Screen Currents and Fields}

We assume that the ground screen shown in figure 8 is perfectly conducting and has zero thickness. The surface current has only a $\rho$ component $J_{\rho}$ which can be written as the sum of the primary surface current $J_{p \rho}$ and an edge-reflected surface current $J_{r \rho}$ :

$$
J_{\rho}(\rho)=J_{p \rho}(\rho)+J_{r \rho}(\rho) .
$$

The primary surface current is defined as the surface current for an infinite ground plane, and it is orthogonal to the primary tangential magnetic field:

$$
J_{p \rho}(\rho)=-\left.H_{p \phi}\right|_{z=0} .
$$

By image theory, the primary tangential magnetic field of the monopole is equal to that of the dipole in free space. That magnetic field expression is well known [6], and the low-frequency (induction field) expression is

$$
\left.H_{p \phi}\right|_{z=0}=\frac{I_{0}}{2 \pi h \rho}\left(\sqrt{\rho^{2}+h^{2}}-\rho\right) .
$$

So the primary surface current on the ground screen is

$$
J_{p \rho}(\rho)=\frac{-I_{0}}{2 \pi h \rho}\left(\sqrt{\rho^{2}+h^{2}}-\rho\right), \quad 0 \leq \rho \leq a .
$$

The primary surface current at the edge of the ground screen $(\rho=a)$ is

$$
J_{p \rho}(a)=\frac{-I_{0}}{2 \pi h a}\left(\sqrt{a^{2}+h^{2}}-a\right) .
$$

The reflected surface current is unknown, but we will use the following approximate expression for an electrically small ground screen [12]

$$
J_{r \rho}(\rho)=-J_{p \rho}(a) \frac{\rho}{a}, \quad 0 \leq \rho \leq a .
$$

This expression has the desirable properties that it causes the total surface current to become zero at the edge of the ground screen $(\rho=a)$ and it does not affect the continuity of the current 
flowing between the monopole and the ground screen at the origin $(\rho=z=0)$. The first property eliminates the ring charge at the edge of the ground screen, and the second property avoids having a point charge at the junction of the monopole and the ground screen.

The surface charge density $q_{s}$ on the ground screen can be determined by taking the surface divergence of the ground screen surface current

$$
q_{s}=\frac{-1}{j \omega} \nabla_{s} \cdot J=\frac{-1}{j \omega} \frac{1}{\rho} \frac{\partial}{\partial \rho}\left(\rho J_{\rho}\right),
$$

where $J_{\rho}$ is given by eqs (36), (39), and (41). Substitution of $J_{\rho}$ into eq (42) yields

$$
q_{s}=\frac{I_{0}}{j \omega 2 \pi h}\left[\frac{1}{\sqrt{\rho^{2}+h^{2}}}-\frac{1}{\rho}-\frac{2}{a^{2}}\left(\sqrt{a^{2}+h^{2}}-a\right)\right], \quad 0 \leq \rho \leq a .
$$

Strictly speaking, there is also a point charge equal to $I_{0} /(j \omega)$ at $\rho=0$, but we chose to neglect it because it is the negative of the point charge that occurs at the base of the monopole. Both point charges are artifacts of breaking the total antenna current distribution into two parts (the monopole and the ground screen) rather than applying the continuity equation to the entire continuous current distribution.

It is interesting to integrate the surface charge density $q_{s}$ over the ground screen surface to determine the total charge $Q_{s}$ on the ground screen:

$$
Q_{s}=\int_{0}^{2 \pi} \int_{0}^{a} q_{s}(\rho) \rho \mathrm{d} \rho \mathrm{d} \phi .
$$

If we substitute eq (43) into eq (44), the $\phi$ integration contributes a factor of $2 \pi$, and the $\rho$ integration can be carried out analytically to yield

$$
Q_{s}=\frac{-I_{0}}{j \omega} .
$$

For comparison, the total charge $Q_{l}$ on the linear monopole element can be written as a line integral:

$$
Q_{l}=\int_{0}^{h} q_{l}(z) d z=\frac{I_{0}}{j \omega} .
$$

Hence the total charge on the monopole antenna is zero:

$$
Q_{l}+Q_{s}=0 \text {. }
$$


This is the expected result and serves as a consistency check on the approximate theory for the ground screen charge. Thus, at large distances, the monopole antenna radiates as two separated charges of opposite polarity (essentially a dipole antenna).

An accurate calculation of the monopole capacitance $C_{m}$ would require a numerical method, but we can make a rough estimate from limiting cases. For a very large ground screen $(a / h \gg 1)$, image theory can be used to show that the monopole capacitance approaches twice the dipole capacitance [13]

$$
\lim _{a \rightarrow \infty} C_{m}=2 C_{d},
$$

where $C_{d}$ is given by eq (8). Method-of-moments calculations [12] show that the dipole capacitance decreases as the ground screen radius decreases. (For $a=0, C_{m}=0$.) We are not interested in very small ground screens because the purpose of using a monopole antenna is to shield the field in the back direction. To estimate the monopole capacitance, it is useful to study the charge distribution on the ground screen. A radial charge density, $q_{s} \equiv 2 \pi \rho q_{s}$, where $q_{s}$ is given by eq (43), is the more relevant quantity because its integral over $\rho$ gives the total charge:

$$
\int_{0}^{a} q_{s \rho} \mathrm{d} \rho=Q_{s}
$$

The radial charge densities are shown in figure 10 for an infinite screen $(a=\infty)$ and a screen of practical size $(a=h)$. Because the charge density for the finite-size screen is more concentrated, its capacitance will be smaller. A rough assumption based on figure 10 is that the monopole capacitance for $a \approx h$ is approximately equal to the dipole capacitance (which is half the value for an infinite screen). The precise value of $C_{m}$ will turn out not to be important in the detection application.

The electric field of the monopole antenna is important in the detection application, and the electric field of the vertical monopole element has already been given. To determine the electric field of the ground screen, the scalar potential $\Phi_{s}$ due to the ground screen charge can be written as the following surface integral

$$
\Phi_{s}=\frac{1}{2 \pi \varepsilon_{0}} \int_{0}^{\pi} \int_{0}^{a} \frac{q_{s}\left(\rho^{\prime}\right)}{R_{s}} \rho^{\prime} \mathrm{d} \rho^{\prime} \mathrm{d} \phi^{\prime}
$$

where $R_{s}=\sqrt{\rho^{2}+\rho^{\prime 2}-2 \rho \rho^{\prime} \cos \phi^{\prime}+z^{2}}$. The geometry is shown in figure 11. Since $\Phi_{s}$ is independent of $\phi$, we have chosen to evaluate it at $\phi=0$. We have also made use of the property that the integrand is even in $\not \partial$. The electric field $\boldsymbol{E}_{s}$ of the ground screen is given by the gradient

$$
E_{s}=-\nabla \Phi_{s}=-\hat{\rho} \frac{\partial \Phi_{s}}{\partial \rho}-\hat{z} \frac{\partial \Phi_{s}}{\partial z} .
$$

Thus the $\rho$ and $z$ components of the electric field are 


$$
E_{s \rho}=\frac{1}{2 \pi \varepsilon_{0}} \int_{0}^{\pi} \int_{0}^{a} \frac{\left(\rho-\rho^{\prime} \cos \phi^{\prime}\right) q_{s}\left(\rho^{\prime}\right)}{R_{s}^{3}} \rho^{\prime} \mathrm{d} \rho^{\prime} \mathrm{d} \phi^{\prime}
$$

and

$$
E_{s z}=\frac{1}{2 \pi \varepsilon_{0}} \int_{0}^{\pi} \int_{0}^{a} \frac{z q_{s}}{R_{s}^{3}} \rho^{\prime} \mathrm{d} \rho^{\prime} \mathrm{d} \phi^{\prime}
$$

The $\not \prime$ integrals in eqs (50), (52), and (53) can be identified as elliptic integrals [14] that cannot be expressed in terms of elementary functions. Probably the best way to evaluate $E_{s \rho}$ and $E_{s z}$ for general observation points would be to perform the double integrations in eqs (52) and (53) numerically. Fortunately, we can evaluate the electric field components on the $z$ axis analytically, and this is sufficient in determining the front-to-back performance of the monopole antenna.

\subsection{On-Axis Field}

The on-axis case $(\rho=0)$ simplifies because $E_{s \rho}=0$ and the integrand in eq (50) is independent of $\not \partial$. Consequently the potential in eq (50) simplifies to

$$
\Phi_{s}=\frac{1}{2 \varepsilon_{0}} \int_{0}^{a} \frac{q_{s}\left(\rho^{\prime}\right)}{\sqrt{\rho^{\prime 2}+z^{2}}} \rho^{\prime} \mathrm{d} \rho^{\prime}
$$

The evaluation of the $\rho$ integration is more involved because of the three terms in $q_{s}$ and the square root in the denominator. However, we can break up $\Phi_{s}$ into three corresponding terms and evaluate each integral analytically:

$$
\Phi_{s}=\Phi_{s 1}+\Phi_{s 2}+\Phi_{s 3}
$$

where

$$
\begin{gathered}
\Phi_{s 1}=\frac{I_{0}}{4 \pi j \omega \varepsilon_{0} h} \ln \left[\frac{\left(\sqrt{\left(a^{2}+h^{2}\right)\left(a^{2}+z^{2}\right)}+2 a^{2}+h^{2}+z^{2}\right)^{1 / 2}}{h+|z|}\right], \\
\Phi_{s 2}=\frac{I_{0}}{4 \pi j \omega \varepsilon_{0} h} \ln \left[\frac{|z|}{a+\sqrt{a^{2}+z^{2}}}\right],
\end{gathered}
$$

and

$$
\Phi_{s 3}=\frac{I_{0}}{2 \pi j \omega \varepsilon_{0} h a}\left(\sqrt{a^{2}+h^{2}}-a\right)\left(|z|-\sqrt{a^{2}+z^{2}}\right) .
$$


The individual terms and the total potential are even in $z$.

Since the $z$ component of the electric field $E_{s z}$ is determined from the negative of the $z$ derivative of eq (55), it can also be written as the sum of three terms:

$$
E_{s z}=E_{s 1 z}+E_{s 2 z}+E_{s 3 z},
$$

where

$$
\begin{gathered}
E_{s 1 z}=\frac{-I_{0}}{4 \pi j \omega \varepsilon_{0} h}\left\{\frac{z\left[\sqrt{\left(a^{2}+h^{2}\right) /\left(a^{2}+z^{2}\right)}+2\right]}{\left.2\left[\sqrt{\left(a^{2}+h^{2}\right)\left(a^{2}+z^{2}\right)+2 a^{2}+h^{2}+z^{2}}\right]-\frac{\operatorname{sgn}(z)}{h+|z|}\right\},}\right. \\
E_{s 2 z}=\frac{-I_{0}}{4 \pi j \omega \varepsilon_{0} h}\left[\frac{1}{z}-\frac{z}{\left(a+\sqrt{a^{2}+z^{2}}\right) \sqrt{a^{2}+z^{2}}}\right], \\
E_{s 3 z}=\frac{I_{0}}{2 \pi j \omega \varepsilon_{0} h a^{2}}\left(\sqrt{a^{2}+h^{2}}-a\right)\left[\frac{z}{\sqrt{a^{2}+z^{2}}}-\operatorname{sgn}(z)\right],
\end{gathered}
$$

and $\operatorname{sgn}(z)$ indicates the sign of $z$. The individual electric field terms in eqs (60) through (62) and hence their sum in eq (59) are odd in $z$.

A good check on eqs (59) through (62) is to let the radius $a$ equal infinity:

$$
\left.E_{s z}\right|_{a=\infty}=\frac{I_{0}}{4 \pi j \omega \varepsilon_{0} h}\left[\frac{1}{z+\operatorname{sgn}(z) h}-\frac{1}{z}\right] .
$$

If we add eqs (35) and (63), we obtain the total electric field of the monopole antenna on the $\mathrm{z}$ axis:

$$
E_{m z}+\left.E_{s z}\right|_{a=\infty}=\frac{I_{0}}{4 \pi j \omega \varepsilon_{0} h}\left\{\begin{array}{l}
\frac{1}{|z-h|}-\frac{2}{z}+\frac{1}{z+h}, z>0 \\
0, z<0
\end{array} .\right.
$$

For positive $z$, this result agrees with the field of a dipole in eq (12). For negative $z$, the field is zero since it is totally shielded by the infinitely large ground screen. In the following section, we take a further look at the difference in the front $(z>0)$ and back $(z<0)$ fields for ground screens of finite radius.

\subsection{Comparison of Front and Back Fields}

On the $z$ axis, the monopole field has only a $z$ component, which is given by 


$$
E_{m z}=\frac{I_{0}}{4 \pi j \omega \varepsilon_{0} h}\left(\frac{1}{|z-h|}-\frac{1}{|z|}\right)
$$

The total field also has only a $z$ component, $E_{t z}=E_{m z}+E_{s z}$, where $E_{m z}$ is given by eq (65) and $E_{s z}$ is given by eq (59). We first look at the field close to the antenna. In the front direction (positive $z$ ), the leading term for $z$ slightly greater than $h$ is the first term in eq (65):

$$
\lim _{z \rightarrow h^{+}} E_{t z}=\frac{I_{0}}{4 \pi j \omega \varepsilon_{0} h(z-h)} .
$$

Hence the field is singular at the tip of the monopole.

In the back direction (negative $z$ ), the singularity for $z \rightarrow 0^{-}$in eq (65) is canceled by the singularity in eq (61). So the leading term for small negative $z$ is a constant:

$$
\lim _{z \rightarrow 0^{-}} E_{t z}=\frac{I_{0}}{2 \pi j \omega \varepsilon_{0} h a^{2}}\left(\sqrt{a^{2}+h^{2}}-a\right) .
$$

For a small ground screen $(a / h<<1)$, eq $(67)$ can be approximated

$$
\lim _{z \rightarrow 0^{-}} E_{z} \approx \frac{I_{0}}{2 \pi j \omega \varepsilon_{0} a^{2}}
$$

For a large ground screen $(a / h>>1)$, the increased shielding reduces the approximation in eq (68) by a factor $h /(2 a)$ :

$$
\lim _{z \rightarrow 0^{-}} E_{t z} \approx \frac{I_{0} h}{4 \pi j \omega \varepsilon_{0} a^{3}} .
$$

The practical implication for a close-in search from eqs (66) and (67) is that the strongest signal will be obtained when the monopole tip approaches the electronic timer.

We look now at $E_{t z}$ on the $z$ axis at large distances $(|z| / h$ and $|z| / a \gg>1)$ from the antenna. If we retain the first two terms in inverse powers of $z$, we obtain the following approximation

$$
E_{t z} \approx \frac{I_{0} h}{4 \pi j \omega \varepsilon_{0}|z|^{3}}\left[1+\frac{C_{4}}{z}\right]
$$

where

$$
C_{4}=\frac{1}{4 h^{2}}\left[\left(a^{2}+4 h^{2}\right) \sqrt{a^{2}+h^{2}}-a^{3}\right] .
$$


The $|z|^{-3}$ term in eq (70) can be identified as the field of a short (Hertzian) dipole of current moment $I_{0} h / 2$. The additional term is due to the extended current distribution on the monopole and the ground screen. For a small screen $(a / h<<1)$, the additonal term is proportional to the monopole length:

$$
C_{4} \approx h \text {. }
$$

For a large screen $(a / h>>1)$, the additional term is proportional to the screen radius:

$$
C_{4} \approx a \text {. }
$$

Since $C_{4}$ is always positive, the $C_{4} / z$ term in eq (70) adds to the leading term in the forward (positive $z$ ) direction, and subtracts in the back (negative $z$ ) direction. So the monopole antenna radiates (or receives) more strongly in the forward direction, but the relative difference between the forward and back radiation decreases as $|z|$ increases.

\section{DETECTION MEASURMENTS}

We performed detection measurements using both a linear dipole receiving antenna and a monopole antenna with a circular ground plane. In both cases, the source was an electronic timer whose radiation characteristics (electric dipole moments) had been measured in a TEM cell [1].

\subsection{Linear Dipole Receiving Antenna}

For our receiving antenna, we constructed a linear dipole of length $1 \mathrm{~m}$. Since the electronic timer radiates a weak, quasi-static electric field, we designed a high-gain, high-inputimpedance amplifier for the detector of the antenna gap voltage. The amplifier matched the high input impedance of the dipole to the $50 \Omega$ impedance of the spectrum analyzer, and the amplifier gain was on the order of $50 \mathrm{~dB}$. To calibrate the combination of the dipole antenna and the amplifier, we used the large NIST TEM cell to generate a known electric field [15] incident on our linear dipole antenna.

Because of the weak timer fields [1], we found it necessary to perform the detection measurements in the underground control room of the NIST open area test site to reduce the competing background noise fields. Even in this environment, the noise fields were higher than expected [16-18]. We performed detection range measurements in both the broadside and end directions from the receiving dipole. Both sets of measurements were performed at a center frequency of $200 \mathrm{kHz}$ with a bandwidth of $10 \mathrm{kHz}$. The noise environment placed limitations on both the center frequency and the bandwidth that had not been a problem with our earlier timer characterization in a shielded TEM cell [1].

The geometry for the broadside measurements is shown in figure 12. The timer was located in the $z=0$ plane with the display face perpendicular to the $z$ axis. The timer dimensions are $6.05 \mathrm{~cm} \times 6.05 \mathrm{~cm} \times 0.85 \mathrm{~cm}$. For this orientation, only the $z$-directed dipole moment $p_{e z}$ couples to the receiving dipole, as indicated by eq (7). Both the measured and theoretical opencircuit voltages are shown in figure 13 as a function of broadside distance $\rho$. The theoretical curve is determined from eqs (7) and (12). The previously measured value of $p_{e z}$ [1] was 
determined to be $25.2 \mathrm{fC} \cdot \mathrm{cm}$ for a bandwidth of $100 \mathrm{kHz}$, rather than $10 \mathrm{kHz}$ as used in the field measurement in figure 13. To convert to an equivalent value of $p_{e z}$ for calculation of the theoretical curve, we make the coherent assumption that we should divide the measured value of $p_{e z}$ by the bandwidth ratio of 10 . This assumption might well be part of the reason for the offset between the measured and calculated curves in figure 13 since we do not really know the detailed spectral characteristics over the full $100 \mathrm{kHz}$ bandwidth. At least the shapes of the two curves as a function of $\rho$ are in good agreement.

The noise floor in figure 13 is determined from the measured open-circuit voltage in the absence of the timer. This noise is due primarily to external sources rather than system noise. From our calibrated antenna and amplifier, we have determined that the equivalent noise spectral density [16] has a value of $0.578 \mu \mathrm{V} /\left(\mathrm{m} \cdot \mathrm{Hz}^{1 / 2}\right)$. As indicated in the Appendix, this value is about a factor of 5 above the expected published value. This is particularly surprising since the underground location is known to provide some shielding from the above-ground fields. This high value is one of the reasons that our detection range is less than expected.

The geometry for the end measurements is shown in figure 14. The same timer was located on the $z$ axis with the display face perpendicular to the $z$ axis. Again, only the $z$-directed dipole moment $p_{e z}$ couples to the receiving dipole, as indicated by eq (7). Both the measured and theoretical open-circuit voltages are shown in figure 15 as a function of $z$. (The end of the dipole is at $z=50 \mathrm{~cm}$.) We again converted the value of $p_{e z}$ measured in the TEM cell for a $100 \mathrm{kHz}$ bandwidth to an estimated value for a $10 \mathrm{kHz}$ bandwidth by dividing by 10 . The theoretical curve for the open-circuit voltage again lies above the measured curve, bringing into question the bandwidth correction. The shapes of the two curves as a function of $z$ are in good agreement.

The noise floor is higher in this case, probably because the receiving antenna was oriented differently to scan the timer position off the end. In this case the equivalent noise spectral density is $1.122 \mu \mathrm{V} /\left(\mathrm{m} \cdot \mathrm{Hz}^{1 / 2}\right)$, about a factor of 10 above the expected published value [16]. Consequently, the detection range is even less than that for the broadside measurement.

\subsection{Monopole Receiving Antenna}

We also constructed a monopole receiving antenna with a ground plane radius of $36 \mathrm{~cm}$ and a monopole length of $35 \mathrm{~cm}$. We used the same high-gain amplifier as we used with the dipole antenna and mounted it below the ground plane. To calibrate the combination of the monopole antenna and the amplifier, we again used the large NIST TEM cell to generate a known electric field [15] incident on the monopole antenna. We again performed the detection measurements in the underground control room of the NIST open area test site to reduce the competing noise fields.

The geometry for these measurements is shown in figure 16. The same timer was located on the $z$ axis with the display face perpendicular to the $z$ axis. Again, only the $z$-directed dipole moment $p_{e z}$ couples to the monopole antenna. Both the measured and theoretical open-circuit voltages are shown in figure 17 as a function of $z$. (The end of the monopole is at $z=35 \mathrm{~cm}$.) The theoretical curve is calculated from eqs (7), (35), and (59). The measurements were performed at a frequency of $200 \mathrm{kHz}$, and we used the same reduced value of the dipole moment to account for the $10 \mathrm{kHz}$ bandwidth. As with the dipole results, the theoretical curve lies well above the measured curve, but the shapes of the curves are in approximate agreement. The noise floor is also shown, and it again represents a noise spectral density that is above the published value [16]. Consequently, the detection range is again very small. 
We have not performed uncertainty analyses for the detection measurements with either the dipole or the monopole receiving antenna. Well established dipole and monopole calibration measurements performed on the open area test site at NIST have expanded uncertainties (with a coverage factor of 2) of approximately $1 \mathrm{~dB}$. These timer detection measurements have larger uncertainties because of the multipath environment of the underground control room and the weak, broadband radiation of the electronic timer.

\section{CONCLUSIONS}

We have developed the theory for the near-field detection of electric-dipole emitters using general receiving antennas. The use of reciprocity theory and the induced EMF method shows that the important quantity for the receiving antenna is the electric field that the same antenna would generate at the emitter location when the antenna is operated in the transmitting mode. The mathematical result is shown in eq (7), and this form is generally easier to evaluate than the open-circuit voltage when the receiving antenna is illuminated by a quasi-static, nonuniform field produced by an electric-dipole emitter. The particular expressions of the transmitted electric field are derived for dipole and monopole antennas and a two-element dipole array. The interesting result for both the dipole and monopole antennas is that the strongest quasi-static electric field is produced off the end of the linear element, rather than in the broadside direction where the far field is maximized.

We constructed both dipole and monopole antennas and performed detection experiments for an electronic timer of the type that could be used in a bomb detonator. The experiments at $200 \mathrm{kHz}$ showed that the timer electric field could be detected, but that the detection range is too short (approximately 10 to $20 \mathrm{~cm}$ ) for practical search applications. Part of the reason for the short detection range is that the background noise fields were larger than expected [16]. This was not an issue in our earlier characterization of electronic timers in TEM cells [1] which provide excellent shielding from external noise sources. Consequently, we conclude that an inspection system (as for baggage) would be feasible using a large TEM cell, but that a timer search method using a portable antenna is probably not feasible in a real-world noise environment.

\section{REFERENCES}

[1] Hill, D.A.; Cavcey, K.H. Dipole moments of weak, electrically small emitters from TEM-cell measurements. Natl. Inst. Stand. Technol. Interagency Report 5079; 1998.

[2] Hill, D.A. Spherical-wave characterization of interior and exterior electromagnetic sources. Natl. Inst. Stand. Technol. Interagency Report 5072; 1997.

[3] Stratton, J.A. Electromagnetic Theory. New York: McGraw-Hill; 1941.

[4] Harrington, R.F. Time-Harmonic Electromagnetic Fields. New York: McGraw-Hill; 1961. 
[5] Monteath, G.D. Applications of the Electromagnetic Reciprocity Principle. Oxford: Pergamon Press; 1973.

[6] Jordan, E.C.; Balmain, K.G. Electromagnetic Waves and Radiating Systems. Englewood Cliffs, NJ: Prentice Hall; 1968.

[7] Park, P.K.; Tai, C.T. Receiving antennas. Ch. 6 in Antenna Handbook (ed., Y.T. and S.W. Lee). New York: Van Nostrand Reinhold Co.; 1988.

[8] Schelkunoff, S.A.; Friss, H.T. Antennas, Theory and Practice. New York: Wiley; 1952.

[9] Ma, M.T. Theory and Application of Antenna Arrays. New York: Wiley, 1974.

[10] Hill, D.A. Gradiometer antennas for detection of long subsurface conductors. $J$. Electromagnetic Waves Applications, 8: 237-248; 1994.

[11] Weiner, M.M. Monopole element at the center of a circular ground plane whose radius is small or comparable to a wavelength. IEEE Trans. Antennas Propagat., 35: 488-495; 1987.

[12] Awadalla, K.H.; Maclean, T.S. Monopole antenna at the center of circular ground plane: input impedance and radiation pattern. IEEE Trans. Antennas Propagat., 27: 151-153; 1979.

[13] Camell, D.G.; Larsen, E.B.; Cruz, J.E.; Hill, D.A. NIST calibration procedure for vertically polarized monopole antennas, $30 \mathrm{kHz}$ to $300 \mathrm{MHz}$. Natl. Inst. Stand. Technol. Technical Note $1347 ; 1991$.

[14] Abramowitz, M.; Stegun, I.A. Handbook of Mathematical Functions. Nat. Bur. Stand. (U.S.) AMS 55; 1964.

[15] Kanda, M.; Orr, R.D. Generation of standard electromagnetic fields in a TEM cell. Nat. Bur. Stand. (U.S.) Technical Note 1319; 1988.

[16] Smith, Jr., A.A. Radio Frequency Principles and Applications. Piscataway, NJ: IEEE Press; 1998.

[17] Characteristics and applications of atmospheric radio noise data. International Radio Consultative Committee Report 322-3: Geneva, Switzerland; 1988.

[18] Fischer, W.H. The radio noise spectrum from e.l.f. to e.h.f. J. Atmos. Terr. Phys., 27; 475-480; 1965. 


\section{APPENDIX: NOISE ENVIRONMENT}

The external noise environment limits the sensitivity of any external electromagnetic field measurement. Noise fields can be expressed in a variety of quantities and units. Since they are all related [16], it is possible to convert noise data to the most convenient quantity. The most convenient noise quantity for our application of electric field measurement is the noise field spectral intensity, which has units of $\mathrm{V} /\left(\mathrm{m} \cdot \mathrm{Hz}^{1 / 2}\right)$. It is normally designated by the symbol $e_{n}$, and the electric field strength $e_{B}$ within a bandwidth $B$ is given by

$$
e_{B}=e_{n} \sqrt{B},
$$

where the bandwidth is given in hertz. Many noise measurements and surveys have been performed over a large range of frequencies $[17,18]$. In our frequency range of interest (approximately $200 \mathrm{kHz}$ to $8 \mathrm{MHz}$ ), both man-made noise and atmospheric noise (due to worldwide lightning) are significant.

Even though both types of noise are highly variable in time and space, a typical value for the noise field spectral intensity at $200 \mathrm{kHz}$ is $e_{n} \approx 0.1 \mu \mathrm{V} /\left(\mathrm{m} \cdot \mathrm{Hz}^{1 / 2}\right)$. Our previous measurements and calculations of electric fields radiated by electronic timers [1] used a $100 \mathrm{kHz}$ bandwidth. So the electric field noise strength at $200 \mathrm{kHz}$ in the same $100 \mathrm{kHz}$ bandwidth is

$$
e_{B} \approx 0.1 \mu \mathrm{V} /\left(\mathrm{m} \cdot \mathrm{Hz}^{1 / 2}\right) \times \sqrt{100 \mathrm{kHz}} \approx 30 \mu \mathrm{V} / \mathrm{m}
$$

Both man-made and atmospheric noise decrease with frequency. However, the timer radiation decreases more rapidly with frequency so that the detection range decreases with frequency. 


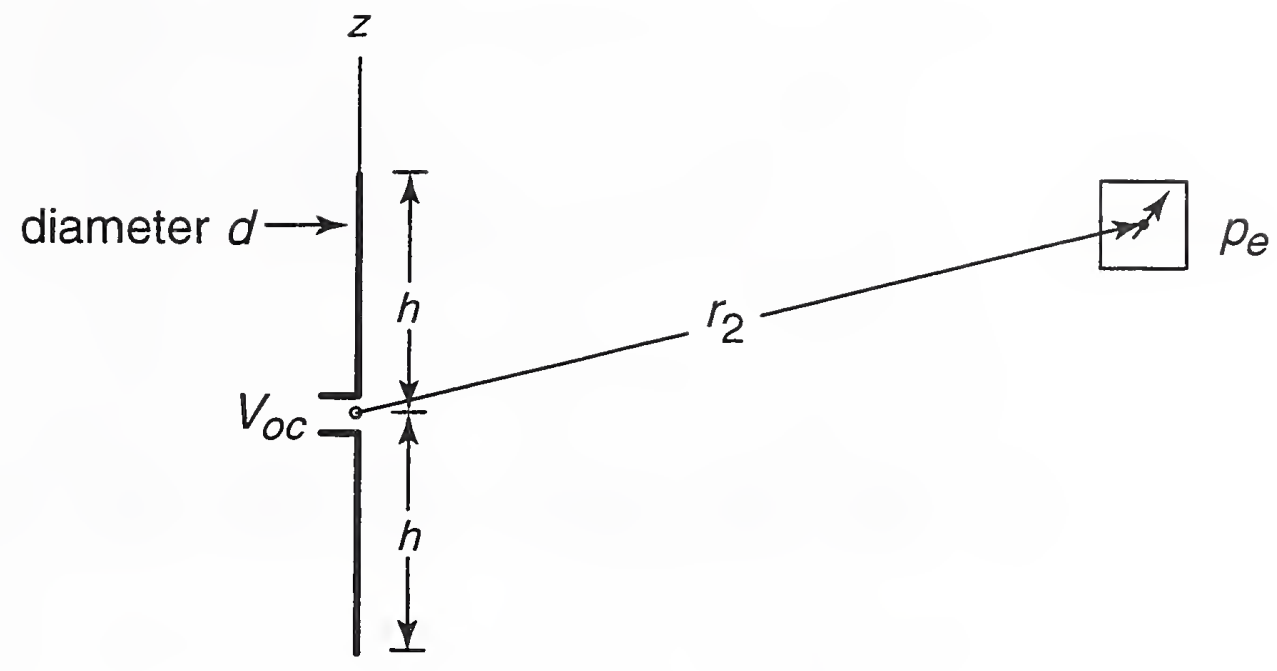

Figure 1. Dipole receiving antenna of length $2 h$ in the presence of an electrically small source of dipole moment $\boldsymbol{p}_{\boldsymbol{e}}$.

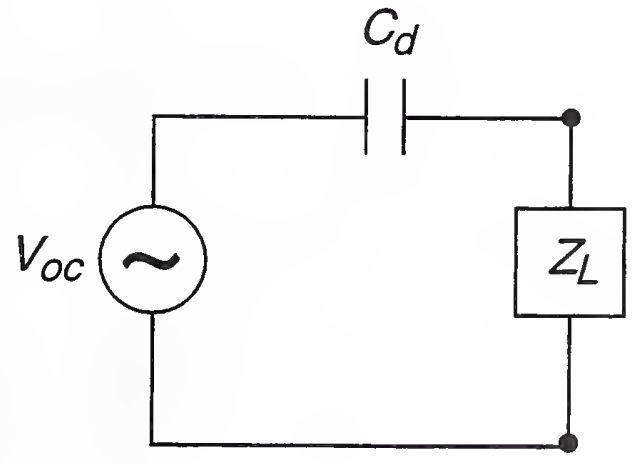

Figure 2. Equivalent circuit for a dipole antenna with capacitance $C_{d}$ and load impedance $Z_{L}$. 


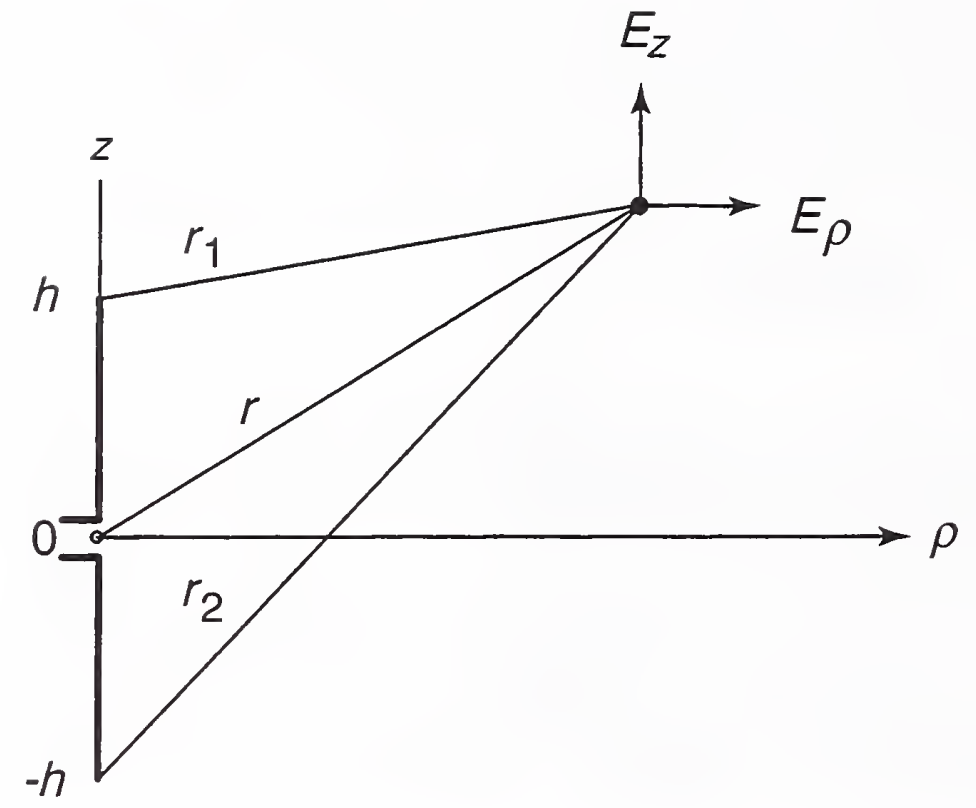

Figure 3. Geometry for the electric fields radiated by a dipole antenna of length $2 h$.

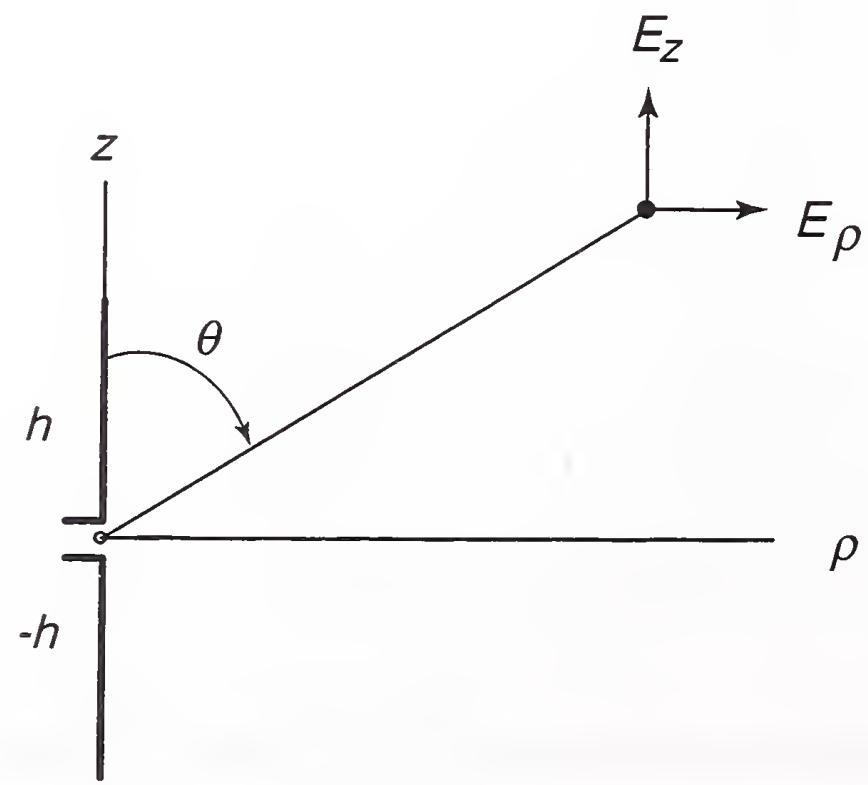

Figure 4. Geometry for the near-field pattern of a dipole antenna. 


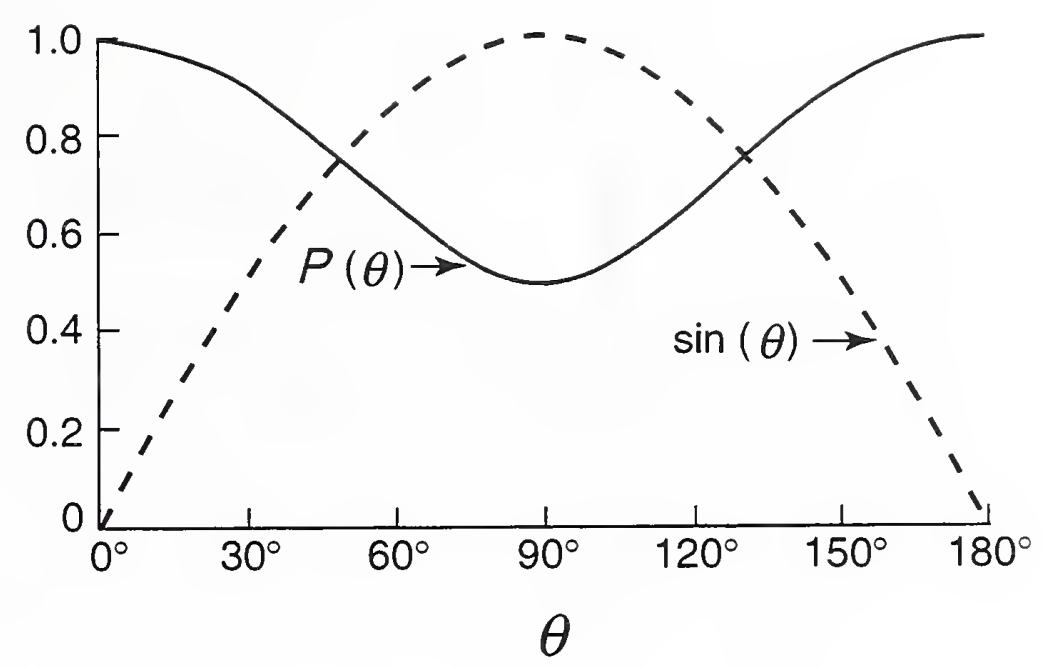

Figure 5. Quasi-static field pattern $P(\theta)$ of a linear dipole compared with $\sin \theta$ far-field pattern.

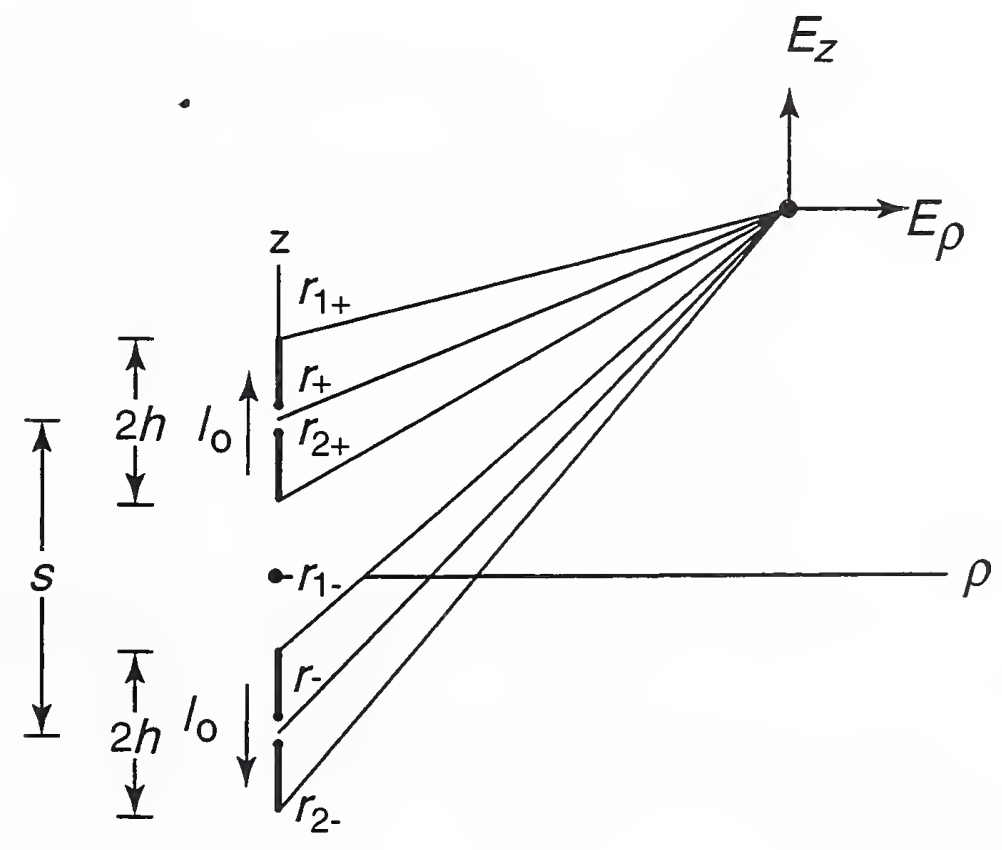

Figure 6. Fields of a two-element, colinear array of dipole antennas fed $180^{\circ}$ out of phase. 


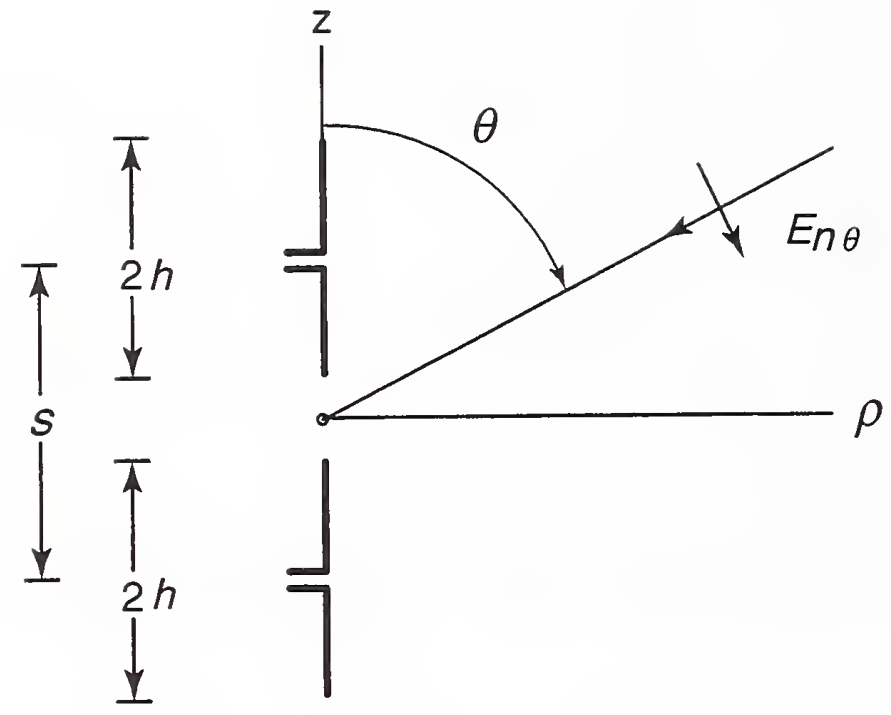

Figure 7. Plane wave field incident on a two-element, linear array of dipole antennas designed for noise cancellation.

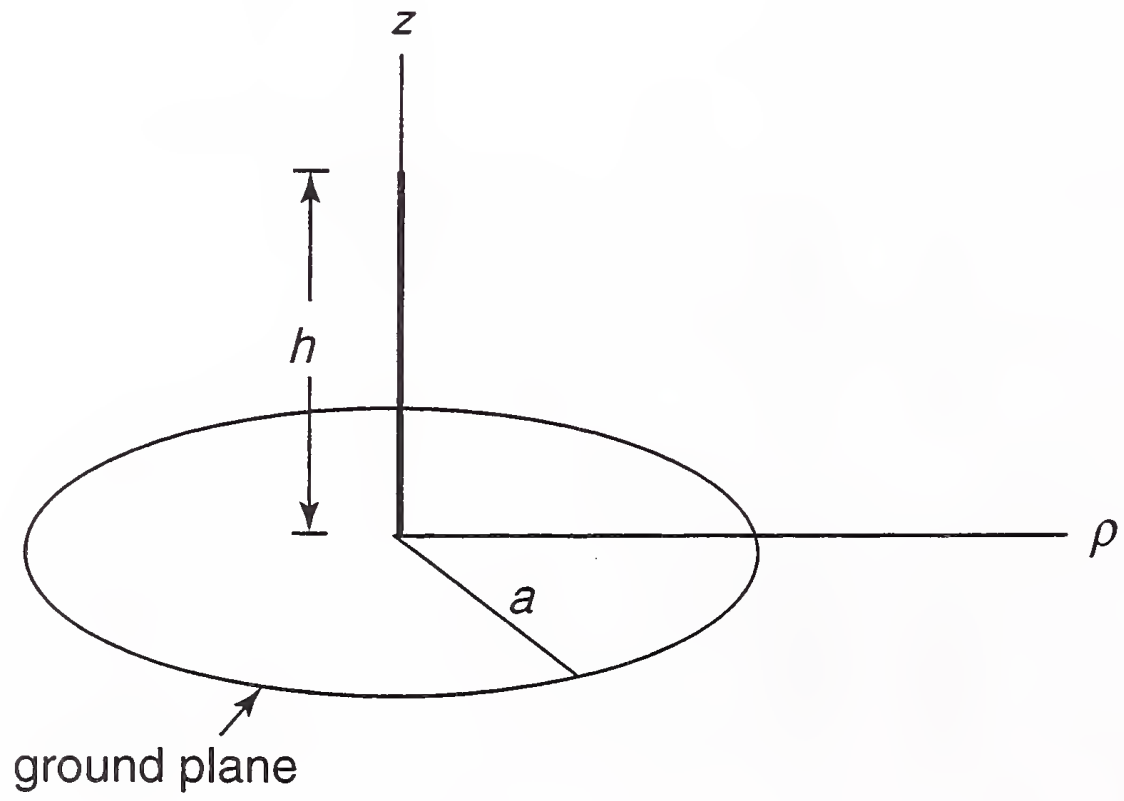

Figure 8. Monopole antenna of length $h$ with a circular ground screen of radius $a$. 


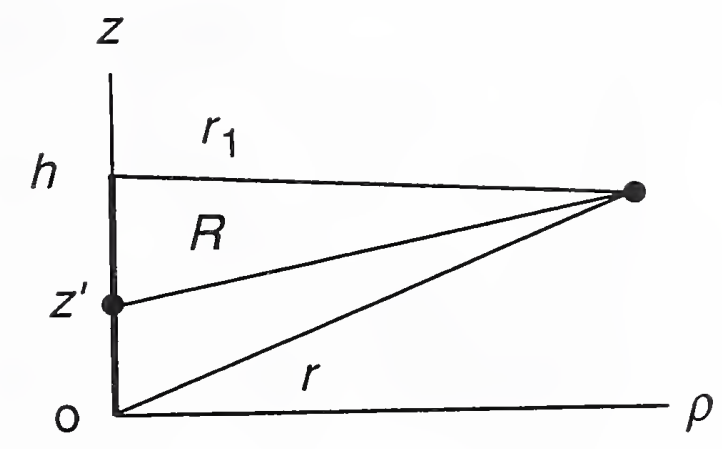

Figure 9. Geometry for fields of a linear monopole of length $h$.

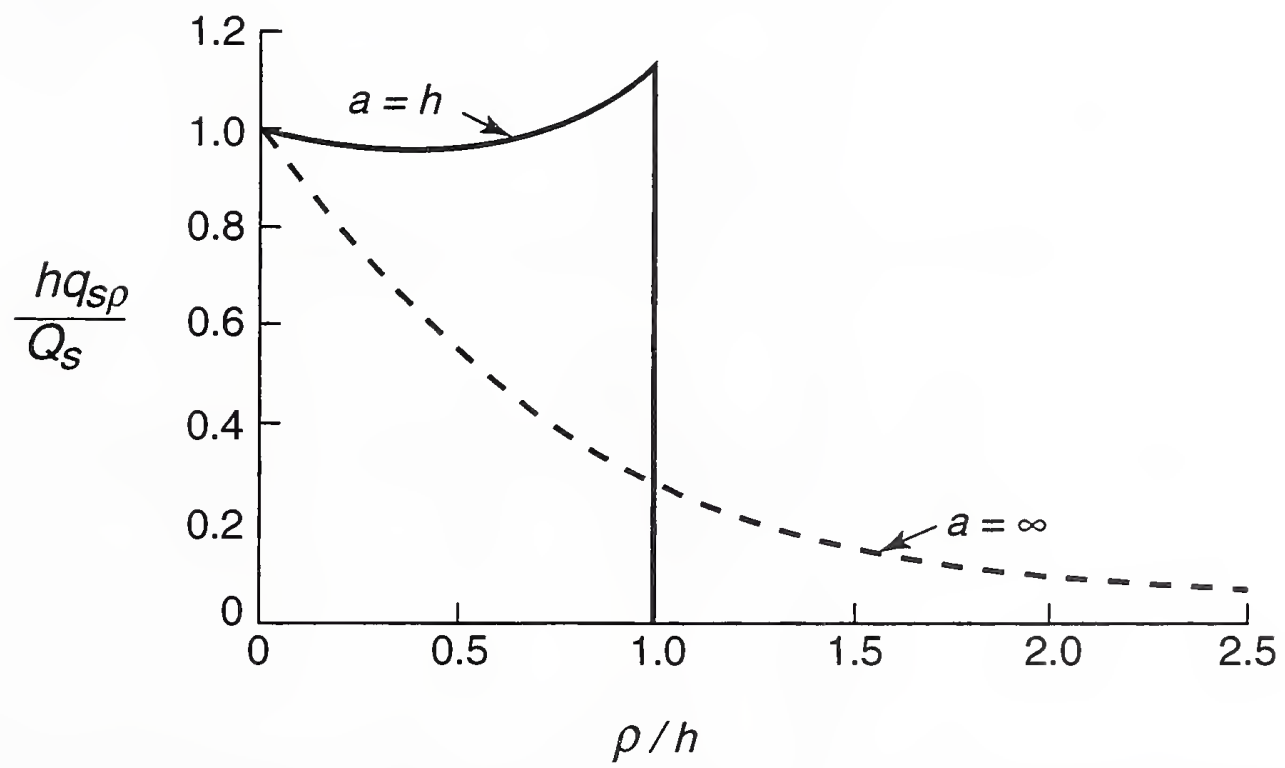

Figure 10. Radial charge density $q_{s \rho}$ on a finite ground screen $(a=h)$ and an infinite ground screen $(a=\infty)$. 


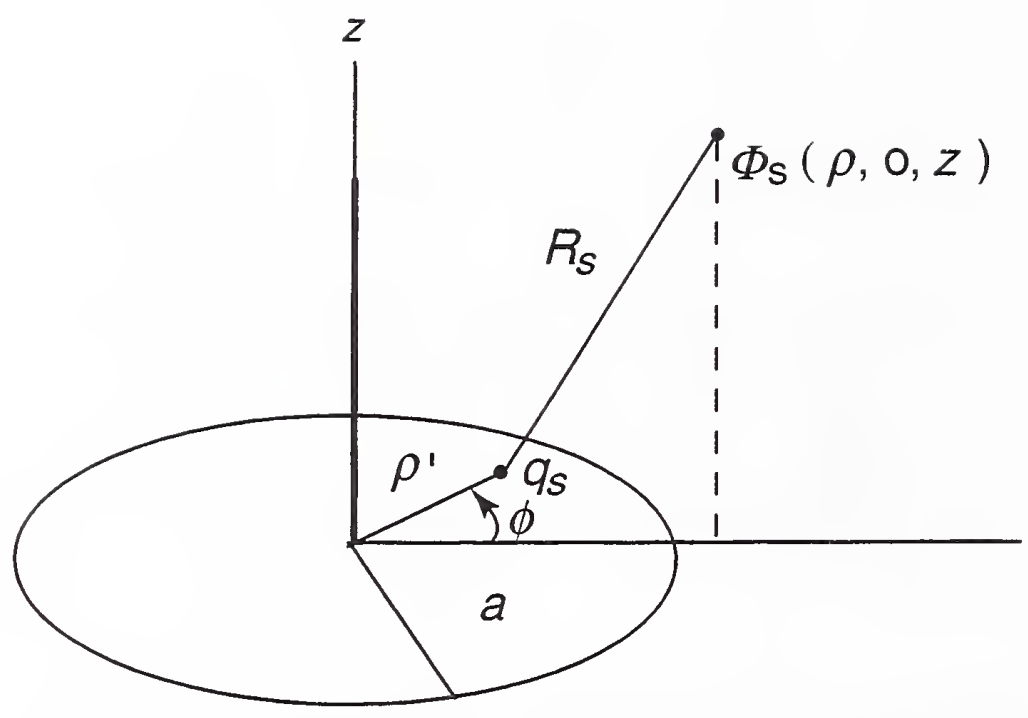

Figure 11. Geometry for the scalar potential $\Phi_{s}$ of the charge $q_{s}$ on a circular ground screen.

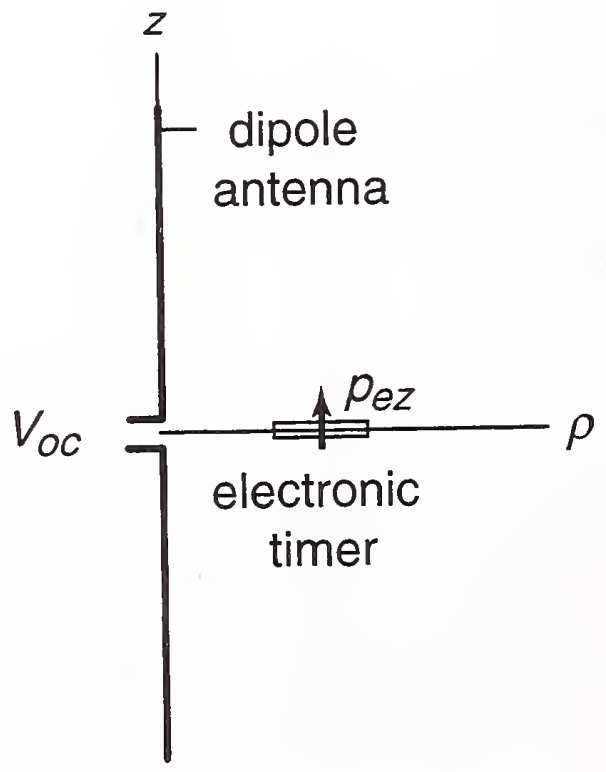

Figure 12. Geometry for an electronic timer located in the broadside direction from a linear dipole receiving antenna. 


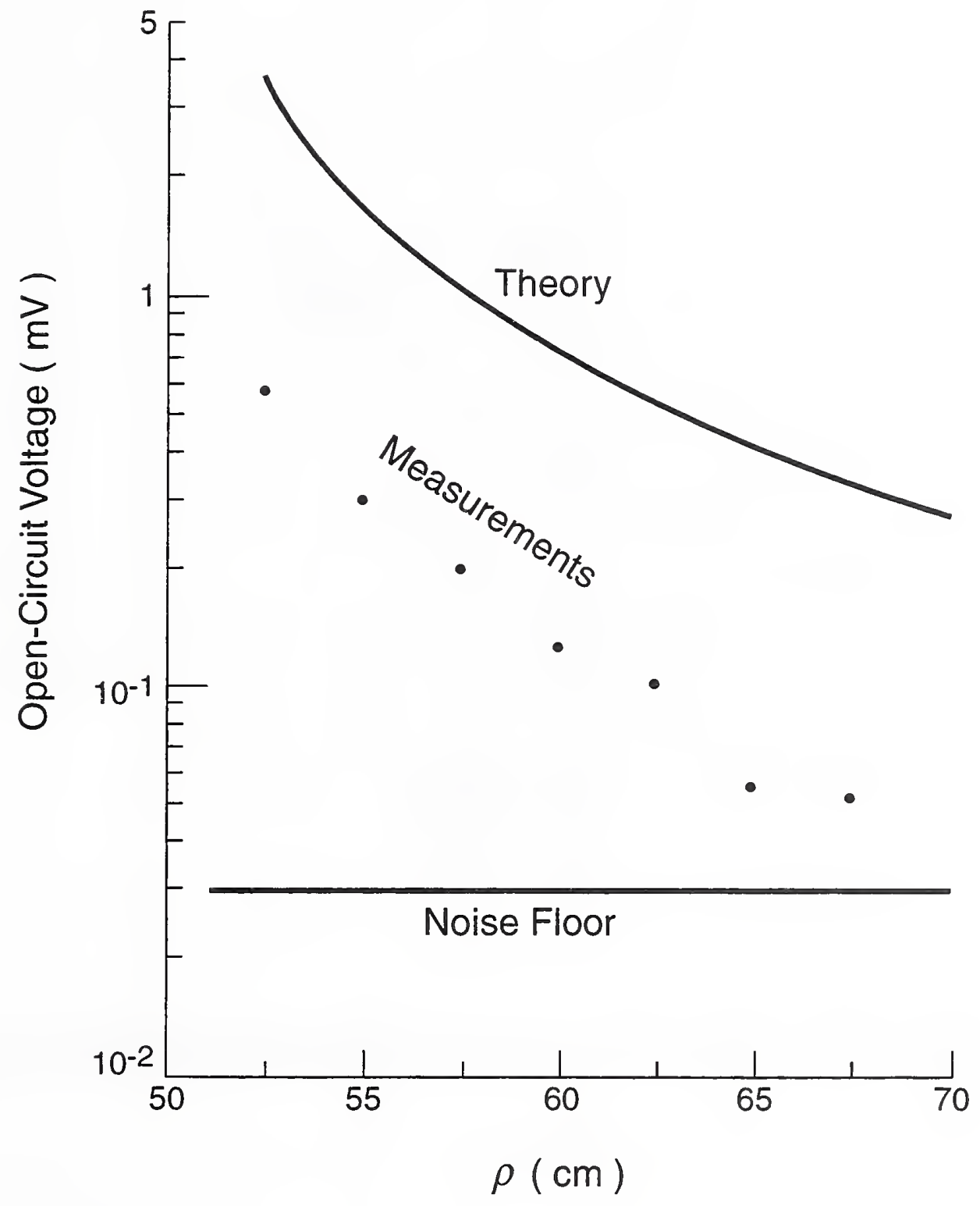

Figure 13. Theory and measurements at $200 \mathrm{kHz}$ for the open-circuit voltage induced in a linear dipole antenna when an electronic timer is located at a distance $\rho$ in the broadside direction. The measured noise floor (in the absence of the timer) is also shown. 


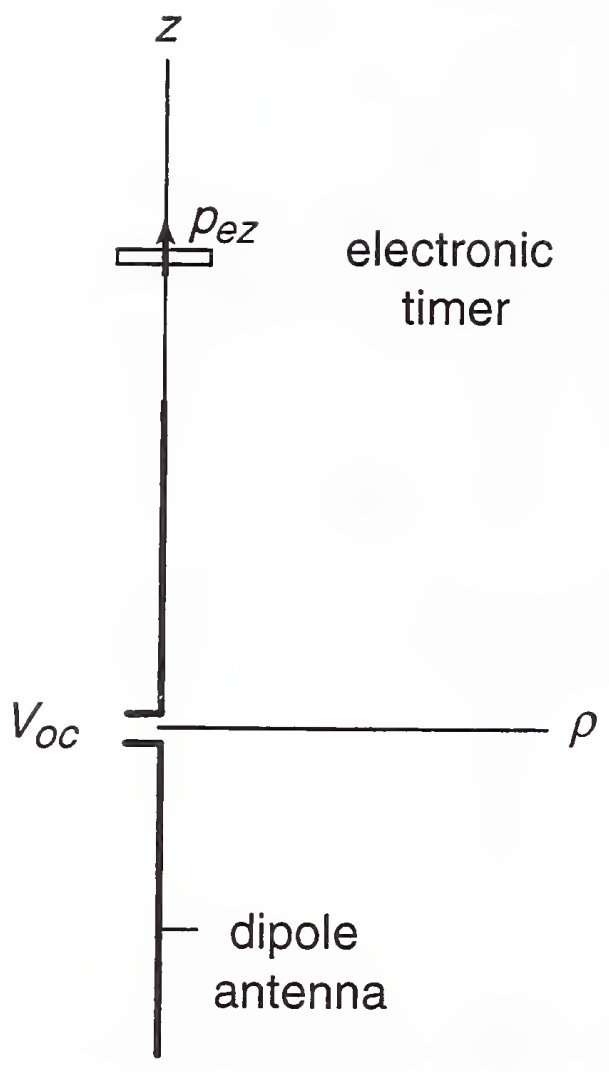

Figure 14. Geometry for an electronic timer located off the end of a linear dipole receiving antenna. 


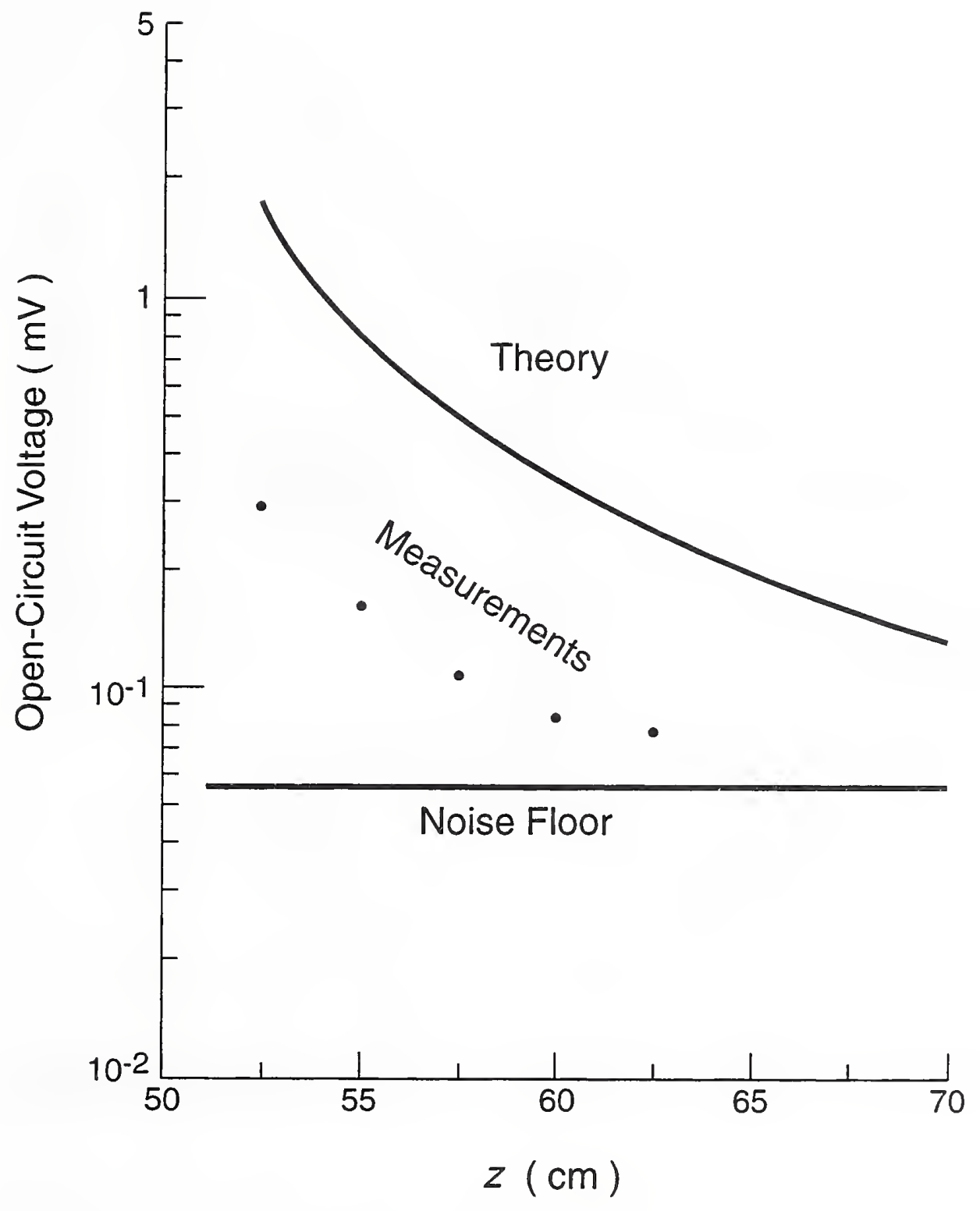

Figure 15. Theory and measurements at $200 \mathrm{kHz}$ for the open-circuit voltage induced in a linear dipole antenna when an electronic timer is located off the end at a distance $z$ from the dipole feed point. The measured noise floor (in the absence of the timer) is also shown. 


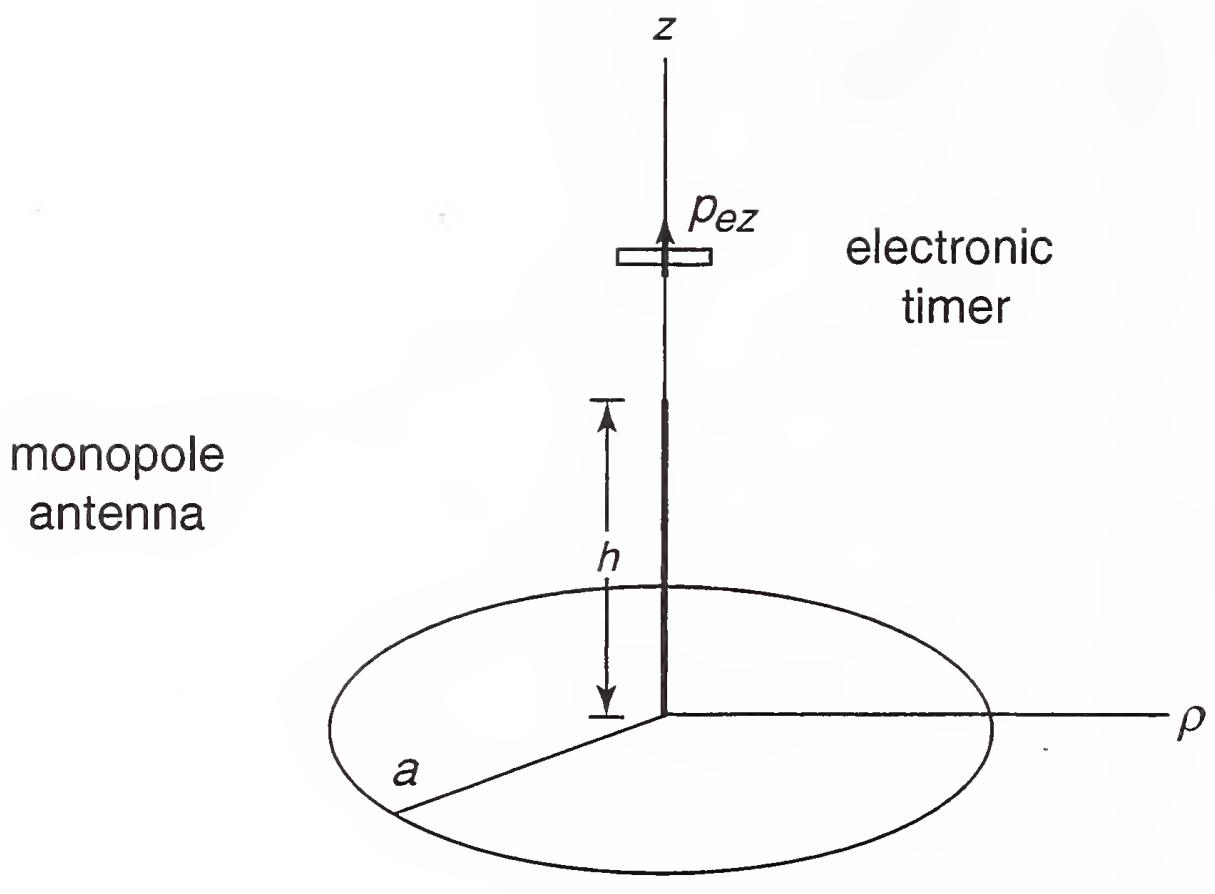

Figure 16. Geometry for an electronic timer located off the end of a monopole receiving antenna. 


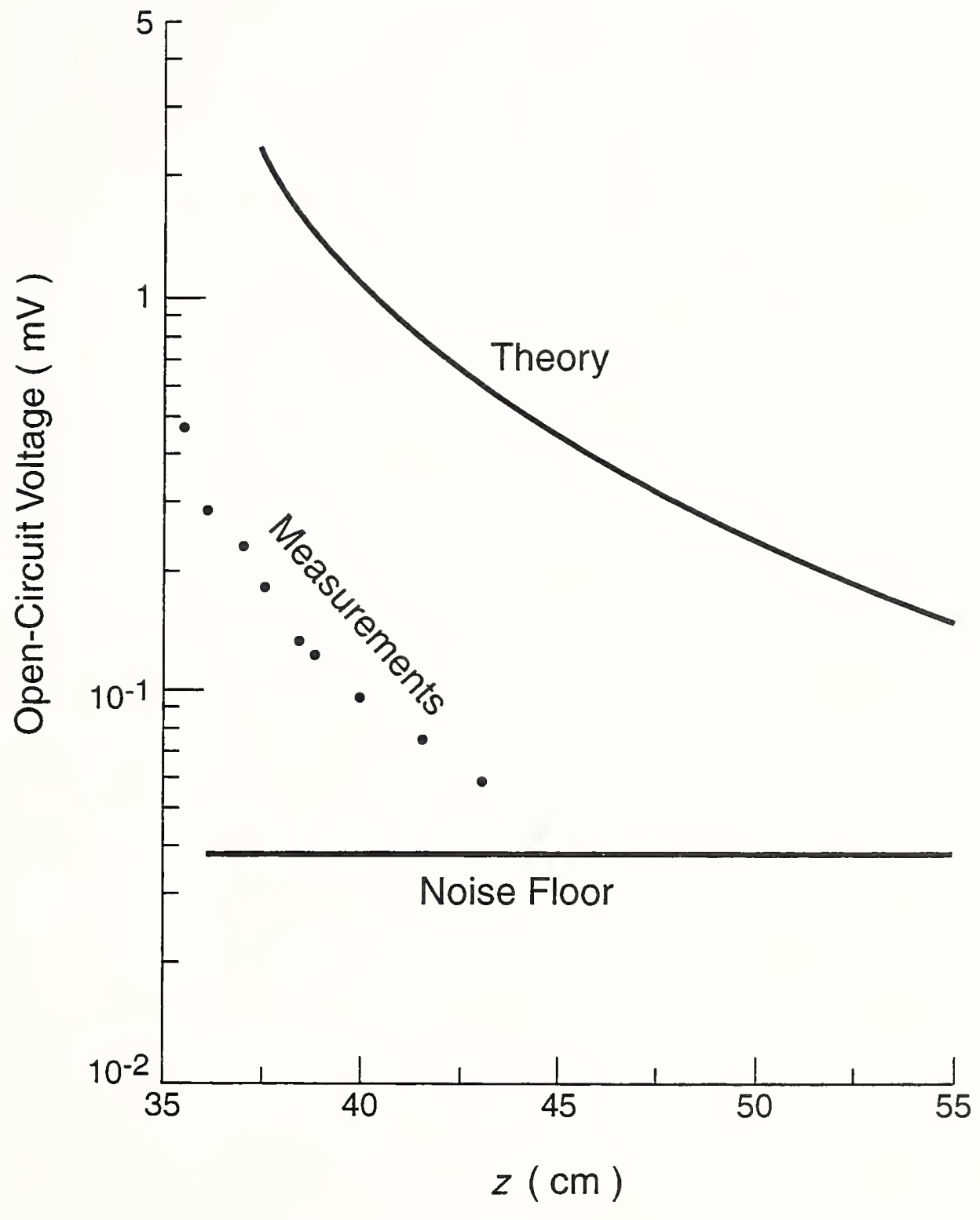

Figure 17. Theory and measurements at $200 \mathrm{kHz}$ for the open-circuit voltage induced in a monopole receiving antenna when an electronic timer is located off the end at a distance $z$ from the monopole feed point. The measured noise floor (in the absence of the timer) is also shown. 
